\title{
Synthesis and Reactivity of Laquinimod, a Quinoline-3-Carboxamide: \\ Intramolecular Transfer of the Enol Proton to a Nitrogen Atom as a Plausible Mechanism for Ketene Formation
}

Karl Jansson, * Tomas Fristedt, Arne Olsson, Bo Svensson, and Stig Jönsson

R\&D Laboratories, Active Biotech Research AB, Box 724, S-220 07 Lund, Sweden

\section{SUPPORTING INFORMATION}

\section{Contents}

Page No.

General methods.

${ }^{1} \mathrm{H}-\mathrm{NMR}$ spectra of compound $\mathbf{1 , 4 , 5 , 6 , 7}$, byproduct in synthesis of $\mathbf{8 , 8}, \mathbf{1 5}, \mathbf{1 7}, \mathbf{1 8} . \quad \mathrm{S} 3-\mathrm{S} 24$

NMR spectra of 2. Assignment of ${ }^{1} \mathrm{H}$ - and ${ }^{13} \mathrm{C}$-shifts obtained from two rotameric $\mathrm{S} 25-\mathrm{S} 30$ forms in $\mathrm{D}_{2} \mathrm{O}+\mathrm{NaOD}$.

Raw data; Impact of the solvent polarity on the reactivity of $\mathbf{2}$. 


\section{General Methods.}

All chemicals used for analytical purposes were of analytical grade. NMR spectra were recorded with an operating frequency of $500.13 \mathrm{MHz}$ for ${ }^{1} \mathrm{H} \mathrm{NMR}$ and $125.8 \mathrm{MHz}$ for ${ }^{13} \mathrm{C}$ NMR. Spectra were recorded at ambient temperature and the shift scale in $\mathrm{CDCl}_{3}$ and $\mathrm{D}_{2} \mathrm{O}$ was referenced to TMS and 3-(trimethylsilyl)-propane sulfonic acid Na-salt (TSPSA) respectively, defined as $0.00 \mathrm{ppm}$ in both ${ }^{1} \mathrm{H}$ - and ${ }^{13} \mathrm{C}$-NMR and to the solvent peak in $\mathrm{D}_{6}$ DMSO, defined as $2.50 \mathrm{ppm}$ for ${ }^{1} \mathrm{H}$ and $39.50 \mathrm{ppm}$ for ${ }^{13} \mathrm{C}$. Signals from two rotameric forms in equilibrium (E/Z-isomerism) were obtained from the sodium salt of 2 in $\mathrm{D}_{2} \mathrm{O}$ and from 18 in $\mathrm{CDCl}_{3}$ and here only the major form is reported. The fact that equilibrium exists is demonstrated in 2D-NOESY spectra by the correlations between the signals of the two forms. Mass spectra were recorded using an ion trap device with an electrospray ion source (ESI) interfaced with an HPLC system. Elemental analysis was performed on an instrument using the flash combustion technique. HPLC analyses were performed using a $\mathrm{C}_{8}$ Symmetry Shield column (Waters) and a solvent gradient system with UV detection at $300 \mathrm{~nm}$.

Solvents for synthesis were used as received or dried with $3 \mathrm{~A}$ molecular sieves when appropriate. Heating of reaction mixtures were performed in oil baths or by using a microwave reactor set to normal absorption level. Sodium sulfate was used for drying of organic extracts prior to concentration on a rotary evaporator at $40^{\circ} \mathrm{C}$. Chromatography was carried out on silica gel 60 using ordinary flash-column chromatography. TLC was performed on silica gel $60 \mathrm{~F}_{254}$ and visualized under UV-light. Solvent systems used for TLC were $\mathrm{CHCl}_{3} / \mathrm{MeOH} / \mathrm{HOAc}$ 100:5:1 or 100:10:1 or n-heptane/EtOAc/HOAc 20:60:3. Precipitates were collected by filtration and dried in vacuum and reaction mixtures were stirred at room temperature unless otherwise noted. 
F2 - Acquisition Parameters

Date__ 980702

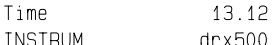

PROBHD $5 \mathrm{~mm}$ GNP $1 \mathrm{H}$

PULPROG $\quad$ zg30

72114
SOLVENT

$\begin{array}{lr}\text { SOLVEN } & \text { COCLI3 } \\ \text { NS } & 16\end{array}$

$\begin{array}{lr}\text { DS } & 0 \\ \text { SWH } & 8992.806 \mathrm{~Hz}\end{array}$

FIDALS $4.124703 \mathrm{~Hz}$

$\begin{array}{ll}\text { AQ } & 4.0095882 \mathrm{sec} \\ \mathrm{FG} & 50\end{array}$

DW $\quad 55.600$ usec

TE $\quad 300.0 \mathrm{~K}$

$\begin{array}{lr}D 1 & 1.00000000 \mathrm{sec} \\ \text { P1 } & 8.50 \mathrm{use}\end{array}$

SF01 500.1342511 MHZ

$\begin{array}{lr}\text { NUC1 } & 1 \mathrm{H} \\ \text { PL1 } & -6.00 \mathrm{~dB}\end{array}$

F2 - Processing parameters

$\begin{array}{lr}\text { SI } & 32768 \\ \text { SF } & 5001300011\end{array}$

WDW

SSB

$\begin{array}{lc}\text { LB } & 0.00 \mathrm{HZ}\end{array}$

PC 1.00

10 NMA plot parameters

$\begin{array}{ll}\text { CX } & 23.00 \mathrm{~cm} \\ \text { cy } & 70.00 \mathrm{~cm}\end{array}$

$\begin{array}{ll}\text { CY } & 70.00 \mathrm{~cm} \\ \text { F1P } & 17.028 \mathrm{com}\end{array}$

F1 $8516.29 \mathrm{~Hz}$

$\begin{array}{ll}F 2 P & -0.173 \mathrm{ppm} \\ F 2 & -86.40 \mathrm{~Hz}\end{array}$

PPMCM $\quad 0.74787 \mathrm{Dom} / \mathrm{c}$

HZCM $\quad 374.02975 \mathrm{~Hz} / \mathrm{cm}$ 
F2 - Acquisition Parameters

Date_ $\quad 980702$

$\begin{array}{ll}\text { Time } & 13.12 \\ \text { INSTRum } & \text { ar } \times 500\end{array}$

PROBHD $5 \mathrm{~mm}$ GNP $1 \mathrm{H}$

2930
72114

$\begin{array}{lr}\text { SOLVENT } & \text { COC13 } \\ \text { NS } & 16\end{array}$

$\begin{array}{lr}\text { DS } & 0 \\ \text { SWH } & 8992.806 \mathrm{~Hz}\end{array}$

FIDRES $\quad 0.124703 \mathrm{~Hz}$

$\begin{array}{ll}\text { AQ } & 4.0095882 \\ \text { FG } & 50\end{array}$

DW $\quad 55.600$ use

$300.0 \mathrm{~K}$
TE
D1

P1 $\quad 8.50$ usec

SF01 500.1342511 MHZ

$\begin{array}{lr}\text { NUC1 } & 1 \mathrm{H} \\ \text { PL1 } & -6.00 \mathrm{~dB}\end{array}$

F2 - Processing parameters

F2 - Processing par ameters
SI 32768

$\begin{array}{lr}\text { SI } & 32768 \\ \text { SF } & 500.1300011 \mathrm{MHZ}\end{array}$

WDW
SSB

$\begin{array}{lc}\text { SSB } & 0.0 \mathrm{~Hz} \\ \text { GB } & 0.00 \mathrm{~Hz}\end{array}$

$\begin{array}{lr}\text { GB } & 0 \\ \text { PC } & 1.00\end{array}$

1D NMR plot parameters

$\begin{array}{ll}\text { CX } & 23.00 \mathrm{~cm} \\ \mathrm{cy} & 70.00 \mathrm{~cm}\end{array}$

$\begin{array}{ll}\text { CY } & 70.00 \mathrm{~cm} \\ \text { F1P } & 7.758 \mathrm{com}\end{array}$

F1 $3879.85 \mathrm{~Hz}$

FशP 6.984 pom

F2 $3492.96 \mathrm{~Hz}$

$\begin{array}{lr}\text { PPMCM } & 0.03363 \mathrm{ppm} / \mathrm{cm} \\ \text { HZCM } & 16.82103 \mathrm{~Hz} / \mathrm{cm}\end{array}$ 
F2 - Acquisition Parameters

Date__ 980701

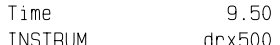

PROBHD $5 \mathrm{~mm}$ GNP $1 \mathrm{H}$

PULPROG $\quad$ zg30

72114
TD
SOLVNT

$\begin{array}{lr}\text { DOLSO } \\ \text { NS } & 16 \\ \text { DS } & 0\end{array}$

$\begin{array}{ll}\text { DS } & 0 \\ \text { SWH } & 10000.000 \mathrm{~Hz}\end{array}$

FIDRES $\quad 0.138669 \mathrm{~Hz}$

$\begin{array}{lr}\text { AQ } & 0.135701 \\ \text { RG } & 100\end{array}$

DW $\quad 50.000$ used

TE

$\begin{array}{ll}\text { D1 } & 1.00000000 \mathrm{sec} \\ \text { P1 } & 8.50 \mathrm{usec}\end{array}$

$\begin{array}{lr}\text { P1 } & 8.50 \mathrm{usec} \\ \text { SF01 } & 500.1340010 \mathrm{MHz} \\ \text { NUC1 } & 1 \mathrm{H}\end{array}$

FL1

F2 - Processing parameters

$\begin{array}{lr}\text { SI } & 32768 \\ \text { SF } & 500.1300054 \\ \text { MH }\end{array}$

WDW EM

$\begin{array}{lr}\text { SSB } & 0 \\ \text { LB } & 0.00 \mathrm{~Hz}\end{array}$

$\begin{array}{lr}\text { GB } & 0 \\ \text { PC } & 1.00\end{array}$

1D NMR plot parameters

$\begin{array}{ll}\text { CX } & 23.00 \mathrm{~cm} \\ \text { CY } & 15.00 \mathrm{~cm}\end{array}$

$\begin{array}{ll}\text { CY } & 15.00 \mathrm{~cm} \\ \text { F1P } & 15.451 \mathrm{com}\end{array}$

F1 $\quad 7727.61 \mathrm{~Hz}$

$\begin{array}{ll}F 2 & -0.095 \mathrm{ppm} \\ \mathrm{F} 2 \mathrm{P} & -47.69 \mathrm{~Hz}\end{array}$

PPMCM $\quad 0.67594 \mathrm{ppm} / \mathrm{c}$

HZCM $\quad 338.05679 \mathrm{~Hz} / \mathrm{cm}$ 
F2 - Acquisition Parameters

$\begin{array}{rr}\text { Date } & 980701 \\ \text { Time } & 950\end{array}$

INSTRUM
PROBHD

PULPROG

$\begin{array}{lr}\text { TD } & 72114 \\ \text { SOLVENT } & \text { DMSO }\end{array}$

$\begin{array}{lr}\text { SOLVENT } & \text { DMSO } \\ \text { NS } & 16 \\ \text { DS } & 0\end{array}$

$\begin{array}{ll}\text { ES } & 0 \\ \text { SWH } & 10000.000 \mathrm{~Hz}\end{array}$

FIDRES - $\quad 0.138669 \mathrm{~Hz}$

$\begin{array}{ll}\text { AQ } & 3.6057501 \\ \text { FG } & 100\end{array}$

DW $\quad 50.000$ usec

TE $3000 \mathrm{~K}$

$\begin{array}{lr}D 1 & 1.00000000 \mathrm{sec} \\ \text { P1 } & 8.50 \mathrm{use}\end{array}$

$\begin{array}{lr}\text { P1 } & 8.50 \text { usec } \\ \text { SF01 } & 500.1340010 \mathrm{MHz}\end{array}$

$\begin{array}{cc}\text { SF01 } & 500.1340010 \mathrm{MP} \\ \text { NUC1 } & 1 \mathrm{H}\end{array}$

FL1

F2 - Processing parameters
ST
32708

$\begin{array}{ll}\text { SI } & 32708 \\ \text { SF } & 500.1300054\end{array}$

WDW EM

$\begin{array}{ll}\text { SSB } & 0.00 \mathrm{~Hz}\end{array}$

$\begin{array}{ll}\mathrm{LB} & 0.00 \\ \mathrm{~GB} & 0 \\ \mathrm{PC} & 1.00\end{array}$

1D NMR plot parameters

$\begin{array}{ll}\text { cX } & 23.00 \mathrm{~cm} \\ \text { cy } & 15.00 \mathrm{~cm}\end{array}$

$\begin{array}{ll}\text { CY } & \\ \text { F1P } & \quad 3.22800000\end{array}$

F1 $\quad 4114.97 \mathrm{~Hz}$

$\begin{array}{ll}\text { F2 } & 3381.29 \mathrm{~Hz}\end{array}$

$\begin{array}{ll}\text { FPMCM } & 0.06378 \mathrm{ppm} / \mathrm{cm} \\ \text { HZCM } & 31.89907 \mathrm{~Hz} / \mathrm{cm}\end{array}$ 
Current Data Parameters

NAME g80630tc

PROCNO

F2 - Acquisition Parameters

Date__ 980630

$\begin{array}{ll}\text { Time } & 14.35 \\ \text { INSTRUM } & \text { an } \times 500\end{array}$

PROBHD $5 \mathrm{~mm}$ GNP $1 \mathrm{H}$

PULPROG $\quad$ zg30

72114
TD

$\begin{array}{lr}\text { NS } & 16 \\ \text { DS } & 0\end{array}$

$\begin{array}{ll}\text { DS } & 0 \\ \text { SWH } & 10000.000 \mathrm{~Hz}\end{array}$

FIDRES $\quad 0.138669 \mathrm{~Hz}$

$\begin{array}{ll}A Q & 3.6057501 \\ \text { AG } & 150\end{array}$

DW $\quad 50.000$ usec

$300.0 \mathrm{~K}$

D1 $1.00000000 \mathrm{sec}$

$\begin{array}{lr}\text { P1 } & 8.50 \mathrm{usec} \\ \text { SF01 } & 500.1340010 \mathrm{MHz}\end{array}$

F2 - Processing parameters

$\begin{array}{lr}\text { SI } & 32768 \\ \text { SF } & 500.1300051 \\ \text { MHZ }\end{array}$

WDW EM

$\begin{array}{lc}\text { SSB } & 0 \\ \text { LB } & 0.30 \mathrm{~Hz}\end{array}$

$\begin{array}{lr}\text { LB } & 0.30 \\ G B & 0 \\ P C & 1.00\end{array}$

1D NMR plot parameters

$\begin{array}{lr}\text { CX } & 23.00 \mathrm{~cm} \\ \mathrm{cr} & 150.00 \mathrm{~cm}\end{array}$

$\begin{array}{ll}\text { CY } & 150.00 \mathrm{~cm} \\ \text { F1P } & 14.085\end{array}$

F1 $7494.32 \mathrm{~Hz}$

F2P $\quad-0.050 \mathrm{ppm}$

Fe $\quad-25.14 \mathrm{~Hz}$

PPMCM $\quad 0.65370 \mathrm{ppm} / \mathrm{cm}$

$326.93332 \mathrm{~Hz} / \mathrm{cm}$ 
Current Data Parameters

NAME

EXPNO

F2 - Acquisition Parameters

Date_ $\quad 980630$

$\begin{array}{ll}\text { Time } & 14.35 \\ \text { INSTRUM } & \text { dr } \times 500\end{array}$

PROBHD $5 \mathrm{~mm}$ GNP $-1 \mathrm{H}$

PULPROG

72114
SOLVENT
COC13

NS 16

SWH $\quad 10000.000 \mathrm{~Hz}$

$\quad 0.138669 \mathrm{~Hz}$

FG $\quad 150$

DW 50.000 use

01
D1 $\quad \begin{array}{r}1.00000000 \mathrm{sec} \\ \mathrm{S}=0.50 \mathrm{usec}\end{array}$

$\begin{array}{lr}\text { P1 } & 8.50 \text { usec } \\ \text { SF01 } & 500.1340010 \mathrm{MHz}\end{array}$

$1 \mathrm{H}$

FL1 $-6.00 \mathrm{~dB}$

F2 - Processing parameters

SI $\quad 32768$

$\begin{array}{lc}\text { WDW } & \text { EM } \\ \text { SSB } & 0 \\ \text { LB } & 0.30 \mathrm{~Hz}\end{array}$

$\begin{array}{lr}\text { GB } & 0.30 \\ \mathrm{~GB} & 1.00\end{array}$

10 NMR plot parameters

$\begin{array}{lr}\text { CX } & 23.00 \mathrm{~cm} \\ \text { cy } & 150.00 \mathrm{~cm}\end{array}$

FIP $\quad-150.00 \mathrm{~cm}$

F1 $\quad \angle 106.80 \mathrm{~Hz}$

$6.940 \mathrm{ppm}$

$3471.12 \mathrm{~Hz}$

$\begin{array}{ll}\text { PPMCM } & 0.05526 \mathrm{ppm} / \mathrm{cm} \\ \text { HZCM } & 27.63842 \mathrm{~Hz} / \mathrm{cm}\end{array}$ 
IH NMR spectrum of 5-chloro-1, 2-dihydro-4-hydroxy-1-methyl-2-oxo-3-quinolinecarboxylic acid in CDCl3.

\begin{tabular}{|c|c|}
\hline Current & ta Parameters \\
\hline NAME & 980703ta \\
\hline EXPNO & 1 \\
\hline PROCNO & 1 \\
\hline$F 2-A C Q$ & sition Parameters \\
\hline Date_ & 980703 \\
\hline Time & 13.53 \\
\hline $\begin{array}{l}\text { INSTRUM } \\
\text { PROBHD }\end{array}$ & \\
\hline $\begin{array}{l}\text { PROBHD } \\
\text { PULPROG }\end{array}$ & 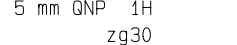 \\
\hline & 72114 \\
\hline SOLVENT & $\mathrm{COC13}$ \\
\hline & 16 \\
\hline DS & 0 \\
\hline SWH & $8992.806 \mathrm{~Hz}$ \\
\hline FIDRES & $0.124703 \mathrm{~Hz}$ \\
\hline & 4.0095882 sec \\
\hline $\mathrm{RG}$ & 300 \\
\hline DW & 55.600 usec \\
\hline DE & 7.50 usec \\
\hline TE & $300.0 \mathrm{~K}$ \\
\hline 01 & $1.00000000 \mathrm{sec}$ \\
\hline $\mathrm{P} 1_{1}$ & 8.50 usec \\
\hline SF01 & $500.1342511 \mathrm{MHz}$ \\
\hline NUC1 & $1 \mathrm{H}$ \\
\hline FL1 & $-6.00 \mathrm{~dB}$ \\
\hline$F_{2}-\operatorname{Pro}$ & ssing parameters \\
\hline SI & 32768 \\
\hline SF & $500.1300124 \mathrm{MHZ}$ \\
\hline WOW & EM \\
\hline SSB & 0 \\
\hline LB & $0.00 \mathrm{~Hz}$ \\
\hline GB & 0 \\
\hline $\mathrm{PC}$ & 1.00 \\
\hline 1D NMA $\mathrm{p}$ & parameters \\
\hline$c x$ & $23.00 \mathrm{~cm}$ \\
\hline$c Y$ & $25.00 \mathrm{~cm}$ \\
\hline F1P & $7.807 \mathrm{ppm}$ \\
\hline$F 1$ & $3904.59 \mathrm{~Hz}$ \\
\hline F2P & $7.161 \mathrm{ppm}$ \\
\hline F2 & $3581.64 \mathrm{~Hz}$ \\
\hline PICM & $\begin{array}{l}0.02808 \mathrm{pm} / \mathrm{cm} \\
1404156 \mathrm{~Hz} / \mathrm{cm}\end{array}$ \\
\hline
\end{tabular}

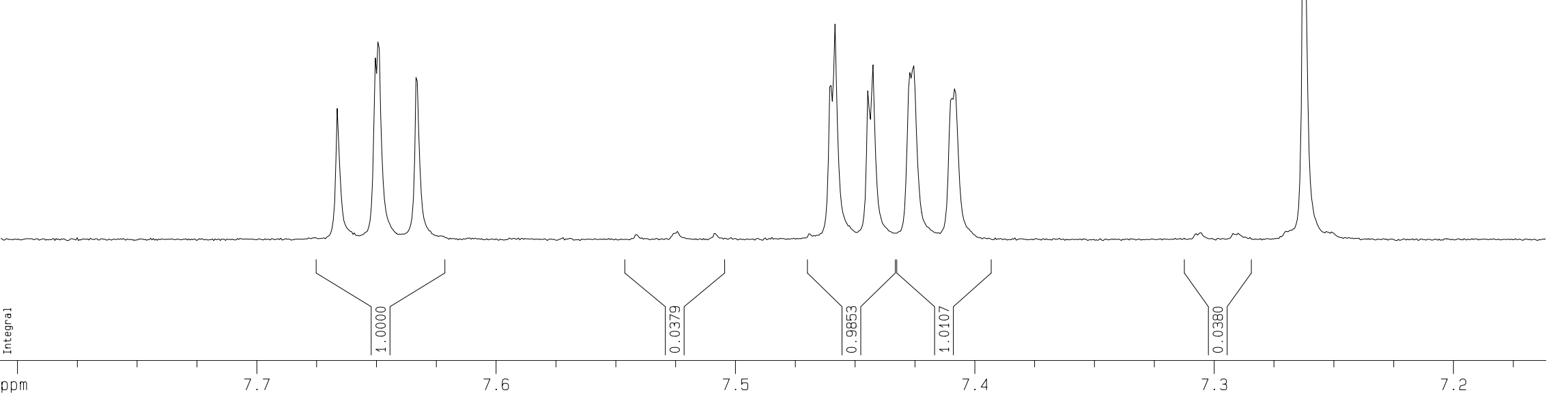


Current Data Parameters NAME $980812 a b$

PROCNO

F2 - Acquisition Parameters

Date__ 980812

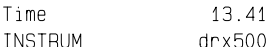

PROBHD $5 \mathrm{~mm} \mathrm{GNP} 1 \mathrm{H}$

PULPROG Z Zg30

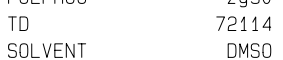

NS $\quad 16$

SWH $\quad 10000.000 \mathrm{~Hz}$

FItAtes $\quad 0.138669 \mathrm{~Hz}$

$\mathrm{AQ} \quad 75$

DW $\quad 50.000$ usec

$300.0 \mathrm{~K}$

$\begin{array}{rr}D 1 & 1.00000000 \mathrm{sec} \\ \mathrm{P1} & 8.50 \mathrm{use}\end{array}$

$\begin{array}{lr}\text { P1 } & 8.50 \text { used } \\ \text { SFO1 } & 500.1340010 \mathrm{MHz}\end{array}$

$500.1340010 \mathrm{MHZ}$
$1 \mathrm{H}$

FL1 $-6.00 \mathrm{~dB}$

F2 - Processing parameters

SI $\quad 32758$

WDW

$\begin{array}{lr}\text { SSB } & 0 \\ L B & 0.30\end{array}$

$\begin{array}{lr} & 0.30 \\ G B & 0 \\ P C & 1.00\end{array}$

10 NMR plot parameters

$\begin{array}{ll}\text { CX } & 23.00 \mathrm{~cm} \\ \text { Cr } & 40.00 \mathrm{~cm}\end{array}$

F1P $\quad 40.00 \mathrm{~cm}$

F1 $\quad 7505.14 \mathrm{~Hz}$

$-0.029 \mathrm{ppm}$

$-14.32 \mathrm{~Hz}$

$\begin{array}{ll}\text { FPMCM } & 0.65370 \mathrm{ppm} / \mathrm{cm} \\ \text { HZCM } & 326.93332 \mathrm{~Hz} / \mathrm{cm}\end{array}$ 


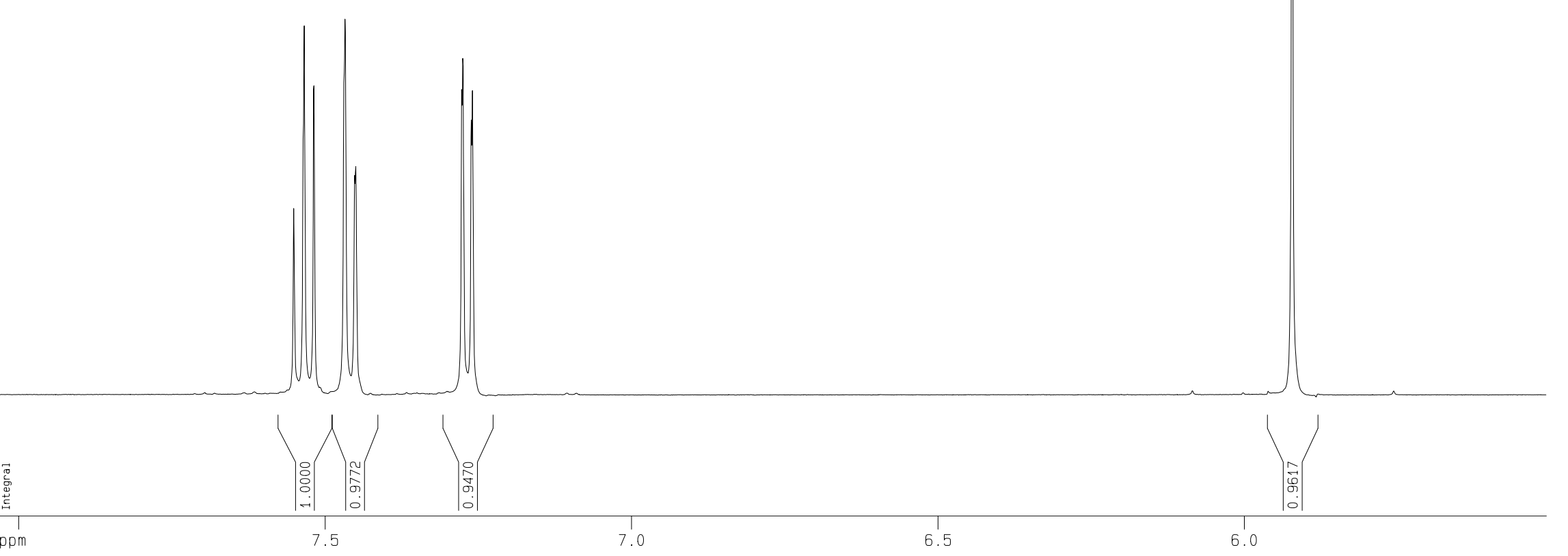


14 NMR spectrum of byproduct in synthesis of 8; 5-Chloro-1, 3-dimethyl-2, 4-dioxo-1, 2, 3, 4-tetrahydro-quinoline-3-carboxylic acid methyl ester in

Curnent Data Parameters

EXPNO

PROCNO

F2 - Acquisition Parameters

Date_ 20030904

$\begin{array}{ll}\text { Time } & 15.50 \\ \text { INSTRUM } & \text { d }\end{array}$

PROBHD $5 \mathrm{~mm}$ QNP $1 \mathrm{H}$

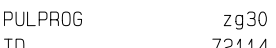

72114
TD

NS $\quad 16$

SWH $\quad 10000.000 \mathrm{~Hz}$

$0.138669 \mathrm{~Hz}$

$A Q$

$\begin{array}{lr}\text { RG } & 200 \\ \text { DW } & 50.000 \text { usec } \\ \text { DE } & 7.50 \text { sec }\end{array}$

TE

D1 3.00000000 sed

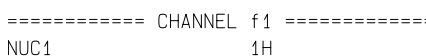

NUC1 $1 \mathrm{H}$

$\begin{array}{ll}\text { P1 } & 9.00 \text { used } \\ \text { PL1 } & -6.00 \mathrm{~dB}\end{array}$

SFO1 $5001340010 \mathrm{MHz}$

2- Processing parameters

SI

SF $\quad 500.1300063 \mathrm{MHZ}$

$\begin{array}{lll}\text { WDW } & \text { EM } \\ \text { SSB } & 0\end{array}$

$\begin{array}{lr}S S B & 0 \\ L B & 0.20 \mathrm{HZ}\end{array}$

$\begin{array}{lr}\text { GB } & 1.00 \\ P C & 1.00\end{array}$

10 NMA plot parameters

CX $23.00 \mathrm{~cm}$

$\begin{array}{ll}\text { CX } & 23.00 \mathrm{~cm} \\ \mathrm{CY} & 25.00 \mathrm{~cm} \\ \mathrm{FH} & 15.000 \mathrm{~cm}\end{array}$

$\begin{array}{ll}F 1 P & 15.000 \mathrm{ppm} \\ F 1 & 7501.05 \mathrm{~Hz}\end{array}$

$\begin{array}{ll}F 1 & 7501.95 \mathrm{~Hz}\end{array}$

$\begin{array}{ll}\text { F2P } & -0.200 \mathrm{ppm} \\ \text { F2 } & -100.03 \mathrm{~Hz}\end{array}$

PPNCM $\quad 0.66087 \mathrm{mpm} / \mathrm{c}$

HZCM $\quad 330.52072 \mathrm{HP} / \mathrm{Cm}$ 
1H NMR spectrum of byproduct in synthesis of 8; 5-Chloro-1, 3-dimethyl-2, 4-dioxo-1, 2, 3, 4-tetrahydro-quinoline-3-carboxylic acid methyl ester in

Current Data Parameters

EXPNO

030904 ta

F2 - Acquisition Parameter

20030904

$\begin{array}{lr}\text { Time } & 15.50 \\ \text { INSTRUM } & \text { dr } \times 500\end{array}$

PROBHD $5 \mathrm{~mm}$ QNP $1 \mathrm{H}$

\begin{tabular}{lr} 
PULPROG & Z930 \\
\hline & 23114
\end{tabular}

72114
SOLVENT

$\begin{array}{lr}\text { NS } & 16 \\ \text { DS } & 2\end{array}$

$\begin{array}{lr}\text { SWH } & 10000.000 \mathrm{~Hz} \\ \text { FIDRES } & 0.138669 \mathrm{~Hz}\end{array}$

$\begin{array}{ll}\mathrm{AQ} & 3.605 / 501 \\ \mathrm{PG} & 200\end{array}$

$\begin{array}{lr}\text { PG } & 200 \\ \text { DW } & 50.000 \mathrm{usec}\end{array}$

7.50 used

[1 $3.00000000 \mathrm{sec}$

$=========$ CHANNEL $f 1===========$
NUC1
$1 \mathrm{H}$

P1 9.00 usec

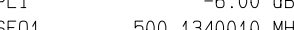

22 - Processing parameters

Fi 2 - Processing

SF $\quad 500.1300063 \mathrm{MHz}$

WDW $\quad$ EM

$\begin{array}{lc}\mathrm{LB} & 0 \\ G B & 0.20 \mathrm{HZ}\end{array}$

1.00

10 NMP plot parameters

$\begin{array}{ll}\text { CX } & 23.00 \mathrm{~cm} \\ \mathrm{CY} & 25.00 \mathrm{~cm} \\ \mathrm{~F} 1 \mathrm{~F} & -7.678 \mathrm{cmm}\end{array}$

$\begin{array}{ll}\text { F1P } & 7.678 \mathrm{pDm} \\ \text { F1 } & 3839.99 \mathrm{~Hz}\end{array}$

FеP

$\quad 3470.94 \mathrm{~Hz}$

PPNCM $\quad 0.03208 \mathrm{ppm} / \mathrm{c}$

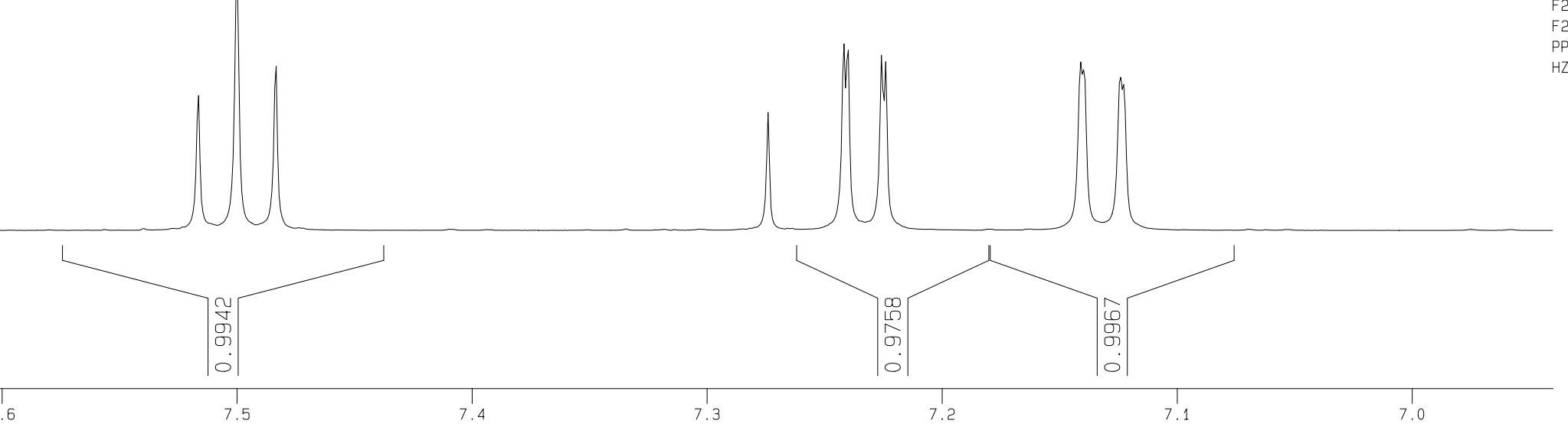


F2 - Acquisition Parameters

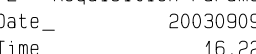

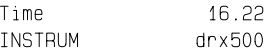

PROBHD $5 \mathrm{~mm}$ GNP $1 \mathrm{H}$

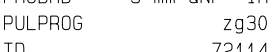

$\begin{array}{ll}\text { SOLVENT } & \text { CDC13 }\end{array}$

16
NS

SWH $\quad 10000.000 \mathrm{~Hz}$

$0.138669 \mathrm{~Hz}$

$\begin{array}{cc}\text { AQ } & 3.6057501 \mathrm{sec} \\ \mathrm{PG} & 200\end{array}$

$\begin{array}{lr}\text { RG } & 200 \\ \text { DW } & 50.000 \text { usec } \\ \text { DE } & 7.50 \text { sec }\end{array}$

TE

D1 3.00000000 sed

$=======-==$ CHANNEL $f 1=$

$\begin{array}{lr}\text { NuC1 } & 1 \mathrm{H} \\ \text { P1 } & 9.00 \mathrm{usec}\end{array}$

500.1340010

2 - Processing parameters

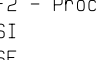

$\begin{array}{ll}\text { SF } & 32760.1300084 \mathrm{MHZ}\end{array}$

WDW $\quad E M$

$\begin{array}{lr}\text { SSB } & 0 \\ \text { LB } & 0.20 \mathrm{HZ}\end{array}$

$\begin{array}{lr}\text { GB } & 0 \\ \text { PC } & 1.00\end{array}$

10 NMF plot parameters

CX $\quad 23.00 \mathrm{~cm}$

$\begin{array}{ll}\text { CX } & 30.00 \mathrm{~cm} \\ \mathrm{CY}\end{array}$

$\begin{array}{lr}F 1 P & 15.979 \mathrm{ppm} \\ F 1 & 7991.50 \mathrm{~Hz}\end{array}$

F2P $-0.168 \mathrm{pD}$

F2 $\quad-84.14 \mathrm{~Hz}$

PPNCM $-0.70205 \mathrm{ppm} /$

$351.11475 \mathrm{~Hz} / \mathrm{c}$ 
F2 - Acquisition Parameters 20030909

$\begin{array}{lr}\text { Time } & 16.25 \\ \text { INSTRUM } & d r \times 500\end{array}$

PROBHD $5 \mathrm{~mm}$ QNP $1 \mathrm{H}$

PULPROG Zg:30

72114
TD

$\begin{array}{lr}\text { NS } & 16 \\ \text { DS } & 2\end{array}$

SWH $\quad 10000.000 \mathrm{~Hz}$

$0.138669 \mathrm{~Hz}$

$\begin{array}{ll}A Q & 3.6057501 \mathrm{sec} \\ \mathrm{PG} & 200\end{array}$

$\begin{array}{lr}\text { PG } & 200 \\ \text { DW } & 50.000 \mathrm{usec}\end{array}$

TE 2007000

D1 $3.00000000 \mathrm{sec}$

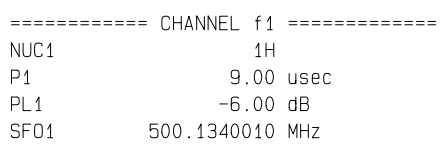

SE01 $500.1340010 \mathrm{dHz}$

F2 - Processing parameters

Fi 2 - Processing

SF $\quad 500.1300084 \mathrm{MHz}$

WDW $\quad$ EM

$\begin{array}{lc}\text { LB } & 0.20 \mathrm{~Hz} \\ \mathrm{~GB} & 0\end{array}$

$\begin{array}{lr}\text { GB } & 1.00 \\ \text { PC } & \end{array}$

10 NMF plot parameters

cX $\quad 23.00 \mathrm{~cm}$

FIP $\quad 30.00 \mathrm{~cm}$

$\begin{array}{ll}\mathrm{F} 1 & 3837.26 \mathrm{~Hz}\end{array}$

$\mathrm{F} 2 \mathrm{P}-7.121 \mathrm{pm}$

F2 $3561.53 \mathrm{~Hz}$

PPNCM - $0.02397 \mathrm{ppm} / \mathrm{c}$

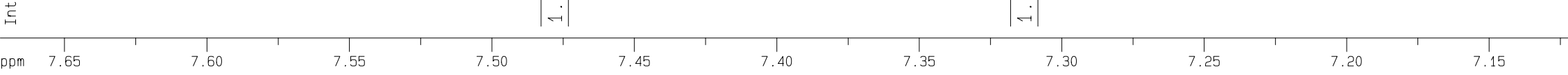


F2 - Acquisition Parameters

$\begin{array}{lr}\text { Date } & 20030905 \\ & \\ \text { Time } & 1555\end{array}$

$\begin{array}{ll}\text { Time } & 15.55 \\ \text { INSTRUM } & \text { dr.5.500 }\end{array}$

PROBHD $5 \mathrm{~mm}$ QNP $1 \mathrm{H}$

PULPROG 7830

72114
TD

NS 16

DS $\quad 10000.000 \mathrm{~Hz}$

$0.138669 \mathrm{~Hz}$

AQ $\quad 3.6057501 \mathrm{sec}$

$\begin{array}{lr}\text { PG } & 200 \\ \text { DW } & 50.000 \text { usec } \\ \text { DE } & 7.50\end{array}$

TE $\quad 7.50$ US

$01 \quad 3.00000000 \mathrm{sed}$

$=========$ CHANNEL $+1=$

P1 9.00 usec

500.1340010 ith

2 - Processing parameters

SI

SF $\quad 500.1300112 \mathrm{MHZ}$

WDW EM

$\begin{array}{lr}\text { SSB } & 0 \\ \text { LB } & 0.20 \mathrm{~Hz}\end{array}$

$\begin{array}{lr}L B & 0.20 \\ G B & 0\end{array}$

D NMR plot parameters

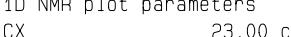

$\begin{array}{ll}\text { CY } & 23.00 \mathrm{~cm} \\ \mathrm{CY} & 25.00 \mathrm{~cm}\end{array}$

$\begin{array}{ll}\mathrm{F} 1 \mathrm{P} & 16.018 \mathrm{ppm}\end{array}$

$80.11 .00 \mathrm{HZ}$
$-0.290 \mathrm{OL}$

$\mathrm{F}_{2}-0.64 \mathrm{~Hz}$

PPNCM

$1145 \mathrm{~Hz} / \mathrm{C}$ 
F2 - Processing parameters

SF $\quad 500.1300112 \mathrm{MHZ}$

WDW

$\begin{array}{lll}L B & 0.20 \mathrm{HZ}\end{array}$

$$
\begin{array}{lr}
G B & 0 \\
P C & 1.00
\end{array}
$$

10 NMA plot parameters

CX $\quad 23.00 \mathrm{~cm}$

CYP $\quad 4.675 \mathrm{cmm}$

F1 $2338.03 \mathrm{~Hz}$

$\begin{array}{lr}F 2 P & 0.872 \mathrm{ppm} \\ \mathrm{F} 2 & 435 \mathrm{~g}\end{array}$

$\begin{array}{ll}435.92 \mathrm{~Hz} \\ \text { PDNCM } & 0.16536 \mathrm{pm} / \mathrm{cm}\end{array}$

$\begin{array}{ll}\mathrm{HZCM} & 0.16536 \mathrm{ppm} / \mathrm{cm} \\ \mathrm{PF} & 82.70058 \mathrm{~Hz} / \mathrm{cm}\end{array}$ 
F2 - Acquisition Parameters

Date__ 980701

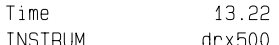

PROBHD $5 \mathrm{~mm}$ GNP $1 \mathrm{H}^{-}$

PULPROG $\quad$ zg30

72114
SOLVENT

$\begin{array}{lr}\text { SOLVENT } & \text { COCl3 } \\ \text { NS } & 16 \\ \text { DS } & 0\end{array}$

$\begin{array}{lr}\text { DS } & 0 \\ \text { SWH } & 10000.000 \mathrm{~Hz}\end{array}$

FIDRES $\quad 0.138669 \mathrm{~Hz}$

$\begin{array}{lr}\mathrm{AQ} & 3.6057501 \mathrm{sec} \\ \mathrm{PG} & 300\end{array}$

DW $\quad 50.000$ usec

TE

$\begin{array}{ll}\text { D1 } & 1.00000000 \mathrm{sec} \\ \text { P1 } & 8.50 \mathrm{ssec}\end{array}$

$\begin{array}{lr}\text { P1 } & 8.50 \mathrm{usec} \\ \text { SFO1 } & 500.1345012 \mathrm{MHz}\end{array}$

SF01 500.1345012 MHz

$\begin{array}{ll}\text { NUC1 } & 1 \mathrm{H} \\ \text { FL1 } & -6.00 \mathrm{~dB}\end{array}$

F2 - Processing parameters

F2 - Processing parameters
SI
32768

$\begin{array}{lr}\text { SI } & 32768 \\ \text { SF } & 500.1300139 \mathrm{MHZ}\end{array}$

$\begin{array}{ll}\text { WDW } & \text { EM } \\ \text { SSB } & 0\end{array}$

$\begin{array}{ll}\text { SSB } & 0.00 \mathrm{~Hz}\end{array}$

$\begin{array}{lr}\text { GB } & 0 \\ \text { PC } & 1.00\end{array}$

10 NMA plot parameters

$\begin{array}{ll}\text { CX } & 23.00 \mathrm{~cm} \\ \text { CY } & 40.00 \mathrm{~cm}\end{array}$

$\begin{array}{lr}\text { CY } & 40.00 \mathrm{~cm} \\ \text { F1P } & 18.814 \mathrm{com}\end{array}$

F1 $\quad 0409.44 \mathrm{~Hz}$

F2P $-0.158 \mathrm{pom}$

F2 $\quad-78.88 \mathrm{~Hz}$

PPMCM $\quad 0.82486 \mathrm{ppm} / \mathrm{c}$

HZCM $\quad 412.53564 \mathrm{~Hz} / \mathrm{cm}$ 
1H NMR spectrum of 5-chloro-3-hydroxy-1-methyl-2, 4-dioxo-1, 2, 3, 4-tetrahydro-quinoline-3-carboxylic acid ethyl-phenyl-amide in CDCl3.

Current Data Parameters 
1H NMR spectrum of 5-chloro-3-hydroxy-1-methyl-2, 4-dioxo-1, 2, 3, 4-tetrahydro-quinoline-3-carboxylic acid

\begin{tabular}{|c|c|}
\hline Current & ta Parameters \\
\hline NAME & $990316 t b$ \\
\hline EXPNO & 1 \\
\hline PROCNO & 1 \\
\hline$F_{2}-A C Q$ & Sition Parameters \\
\hline Date_ & 990316 \\
\hline Time & 11.26 \\
\hline INSTRUM & $d r \times 500$ \\
\hline PROBHD & $5 \mathrm{~mm}$ GNP $1 \mathrm{H}$ \\
\hline PULPROG & zg30 \\
\hline TD & 72114 \\
\hline SOLVENT & DMSO \\
\hline NS & 16 \\
\hline DS & 0 \\
\hline SWH & $10000.000 \mathrm{~Hz}$ \\
\hline FIDRES & $0.138669 \mathrm{~Hz}$ \\
\hline & 3.6057501 sec \\
\hline $\mathrm{FG}$ & 100 \\
\hline DW & 50.000 usec \\
\hline DE & 7.50 usec \\
\hline TE & $297.0 \mathrm{~K}$ \\
\hline D1 & $2.00000000 \mathrm{sec}$ \\
\hline $\mathrm{P}_{1}$ & 8.50 usec \\
\hline SF01 & $500.1340010 \mathrm{MHz}$ \\
\hline NUC1 & $1 \mathrm{H}$ \\
\hline FL1 & $-6.00 \mathrm{~dB}$ \\
\hline$F_{5}-\operatorname{Pro}$ & ssing parameters \\
\hline SI & 32768 \\
\hline $\mathrm{SF}$ & $500.1300096 \mathrm{MHZ}$ \\
\hline WOW & EM \\
\hline SSB & 0 \\
\hline LB & $0.30 \mathrm{~Hz}$ \\
\hline$G B$ & 0 \\
\hline PC & 1.00 \\
\hline 1D NMP p & parameters \\
\hline & $23.00 \mathrm{~cm}$ \\
\hline CY & $25.00 \mathrm{~cm}$ \\
\hline F1P & $7.653 \mathrm{ppm}$ \\
\hline F1 & $3827.39 \mathrm{~Hz}$ \\
\hline F2P & $6.605 \mathrm{ppm}$ \\
\hline F2 & $3303.44 \mathrm{~Hz}$ \\
\hline PPMCM & $0.04555 \mathrm{pm} / \mathrm{cm}$ \\
\hline $\mathrm{HZCM}$ & $22.78060 \mathrm{~Hz} / \mathrm{cm}$ \\
\hline
\end{tabular}

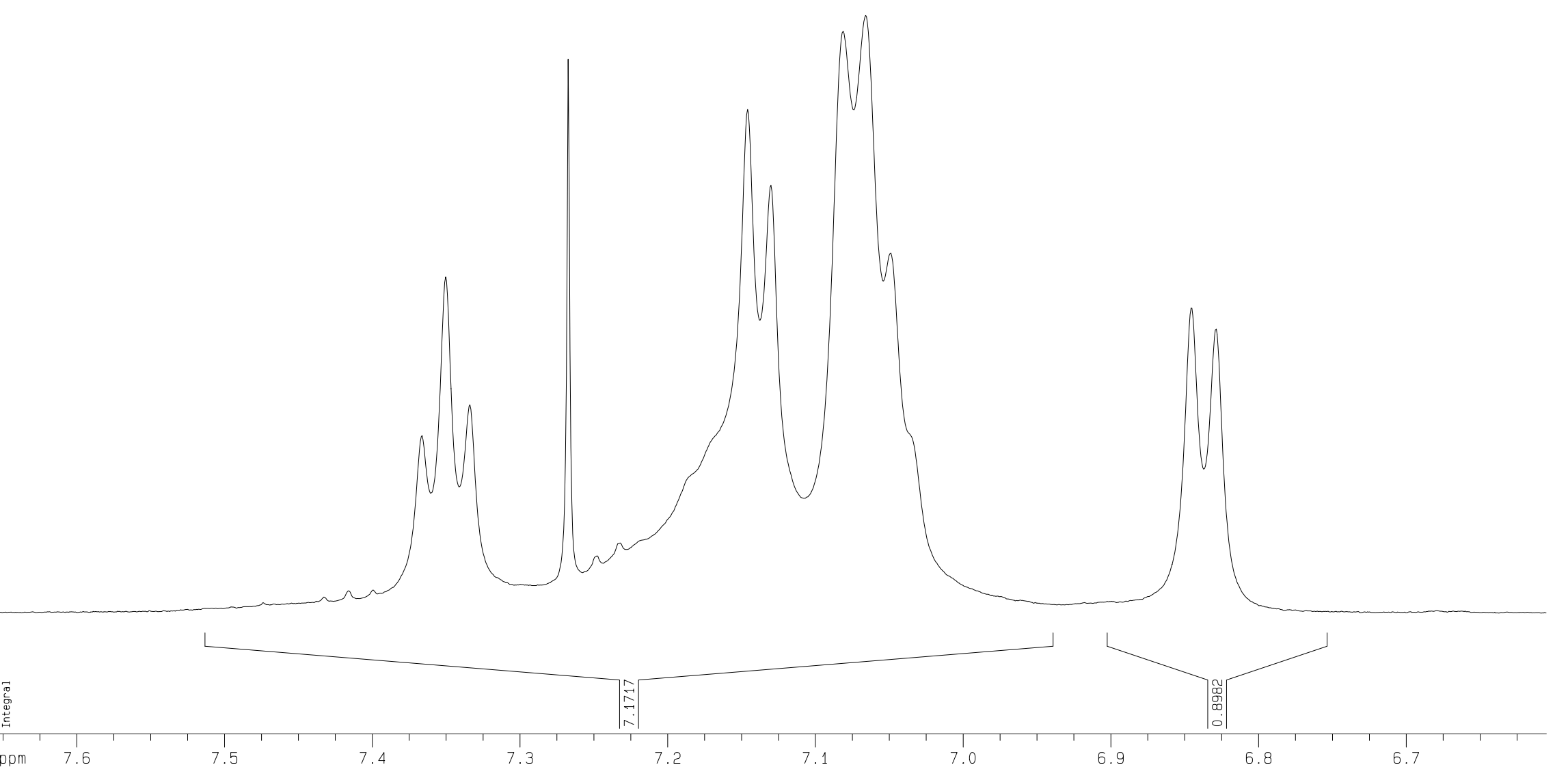


IH NMR spectrum of 5-chloro-3-hydroxy-1-methyl-2, 4-dioxo-1, 2, 3, 4-tetrahydro-quinoline-3-carboxylic acid

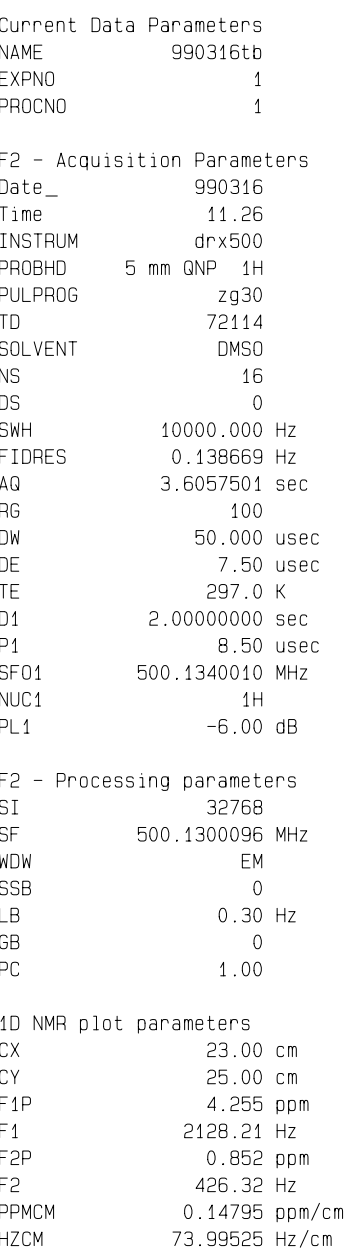


Assignment of NMR-signals for 2 in $\mathrm{D}_{2} \mathrm{O}+\mathrm{NaOD}$ (conc: about $20 \mathrm{mg} / \mathrm{ml}$ ). The listed assignments refer to the structure formula below. Note! Large ${ }^{1} \mathrm{H}$-shift variations are obtained in alkaline $\mathrm{D}_{2} \mathrm{O}$ solution dependent on the concentration of $\mathbf{2}$. Minor ${ }^{1} \mathrm{H}$-shift variations are also seen with NaOD concentration.

\begin{tabular}{|c|c|c|c|c|}
\hline \multicolumn{2}{|c|}{ 13C-shifts } & \multicolumn{2}{|c|}{ 1H-shifts } & \multirow[t]{2}{*}{ Assignment } \\
\hline Major form & Minor form & Major form & Minor form & \\
\hline 173.7 & 175.0 & - & - & (11)-CO-N \\
\hline 173.5 & 174.0 & - & - & $(4)-C=$ \\
\hline 164.2 & 164.8 & - & - & (2)-CO-N \\
\hline 144.4 & 143.4 & - & - & $\left(1^{\prime}\right)-\mathrm{C}=$ \\
\hline 144.3 & 144.8 & - & - & $(9)-C=$ \\
\hline 134.2 & 134.7 & - & - & $(5)-C=$ \\
\hline 132.3 & 132.8 & 6.81 & 7.17 & $(7)-\mathrm{CH}=$ \\
\hline 131.2 & 132.2 & 7.19 & 7.62 & $\left(3^{\prime}, 5^{\prime}\right)-\mathrm{CH}=$ \\
\hline 130.5 & 130.5 & 6.97 & 7.51 & $\left(4^{\prime}\right)-\mathrm{CH}=$ \\
\hline 129.3 & 130.7 & 7.41 & 7.51 & $\left(2^{\prime}, 6^{\prime}\right)-\mathrm{CH}=$ \\
\hline 127.2 & 127.6 & 6.84 & 7.12 & (6)-CH= \\
\hline 121.2 & 121.6 & - & - & $(10)-C=$ \\
\hline 116.3 & 116.8 & 6.53 & 7.01 & $(8)-\mathrm{CH}=$ \\
\hline 111.4 & 109.5 & - & - & $(3)-C=$ \\
\hline 46.7 & 50.1 & \begin{tabular}{|l|}
3.91 \\
3.88
\end{tabular} & $\begin{array}{l}3.63 \\
3.73\end{array}$ & (12)-N- $\underline{C H}_{2}-\mathrm{CH}_{3}$ \\
\hline 32.1 & 32.3 & 3.15 & 3.47 & $(1)-\mathrm{N}-\mathrm{CH}_{3}$ \\
\hline 14.9 & 16.1 & 1.24 & 0.98 & (12)-N-CH$-\underline{C H}_{3}$ \\
\hline
\end{tabular}<smiles>CCN(C(=O)c1c(Cl)c2c(Cl)cccc2n(C)c1=O)c1ccccc1</smiles> 


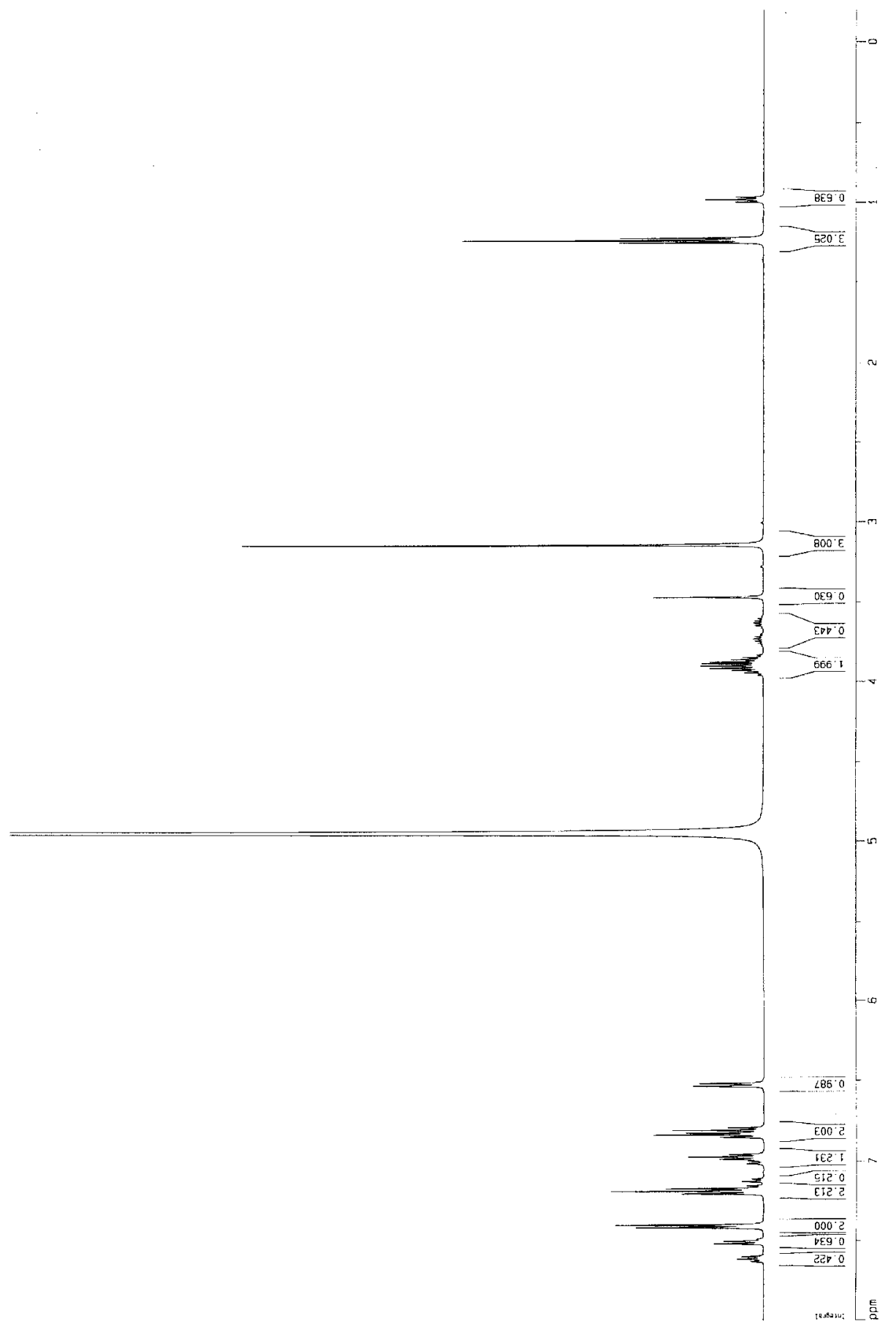

${ }^{1} \mathrm{H}-\mathrm{NMR}$ spectrum of 2 in $\mathrm{D}_{2} \mathrm{O}+\mathrm{NaOD}$ in a concentration of about $20 \mathrm{mg} / \mathrm{ml}$. 

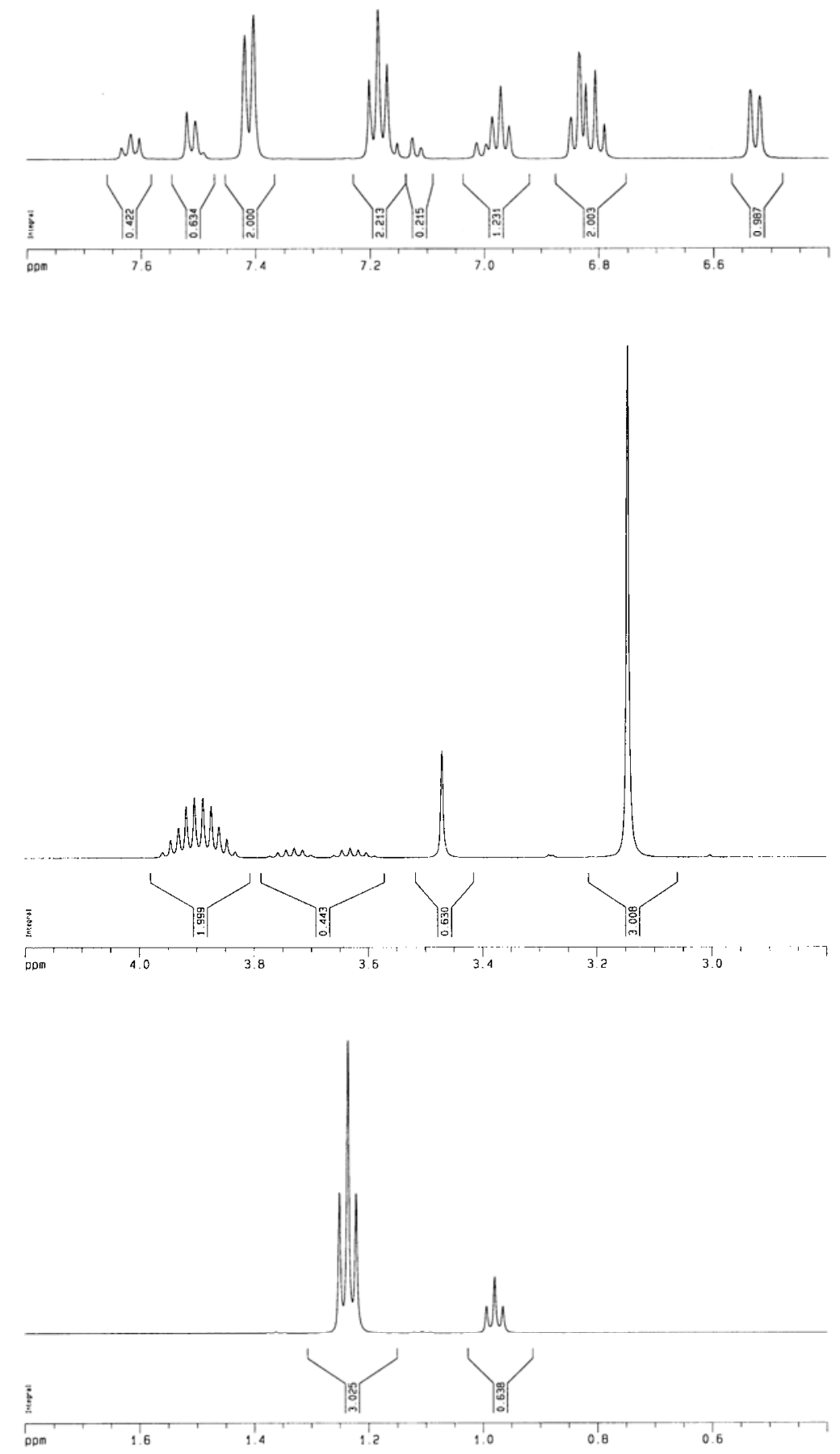

Expansions of ${ }^{1} \mathrm{H}-\mathrm{NMR}$ spectrum of 2 in $\mathrm{D}_{2} \mathrm{O}+\mathrm{NaOD}$ in a concentration of about $20 \mathrm{mg} / \mathrm{ml}$. 


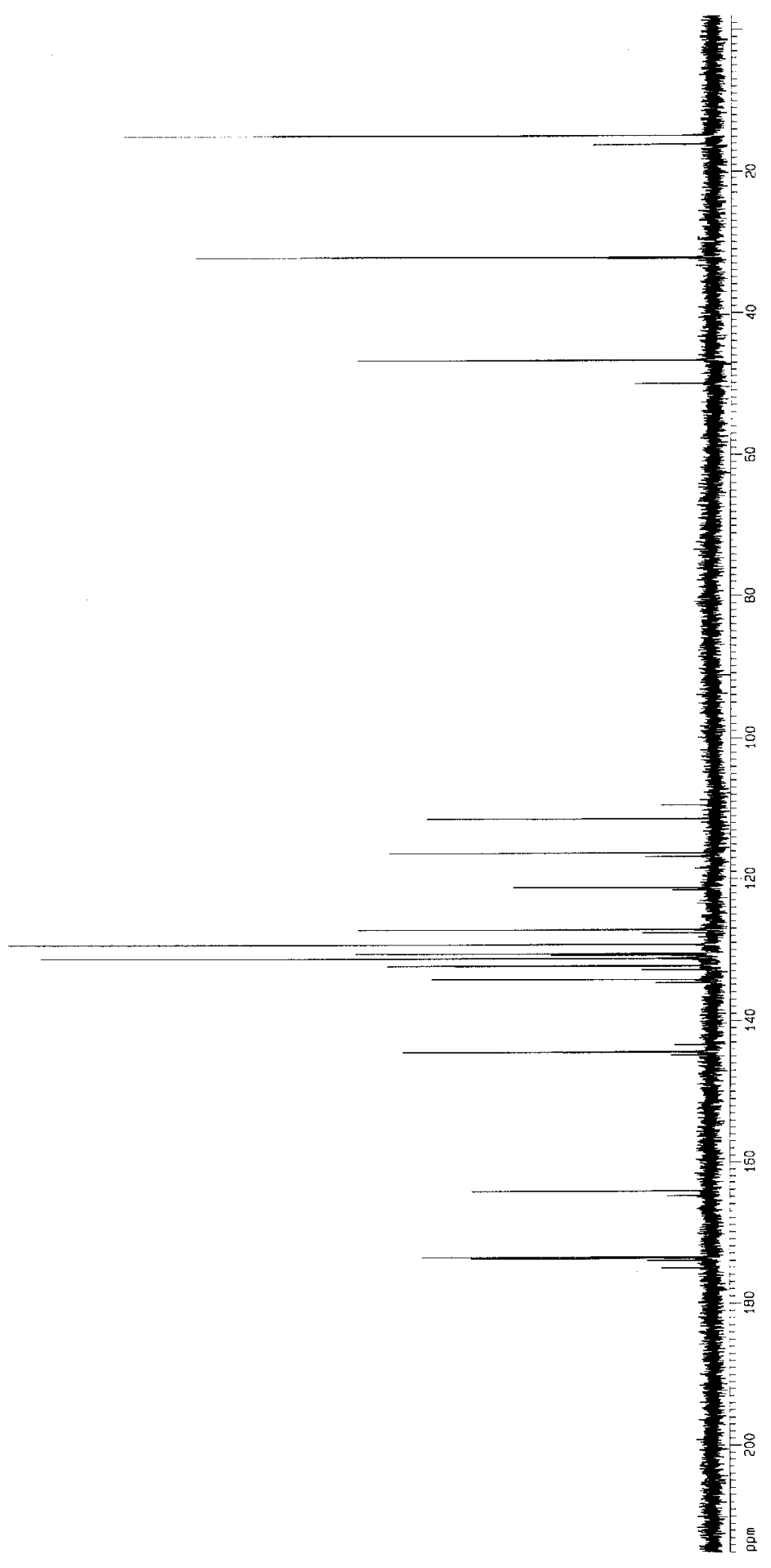

${ }^{13} \mathrm{C}-\mathrm{NMR}$ spectrum of 2 in $\mathrm{D}_{2} \mathrm{O}+\mathrm{NaOD}$. 


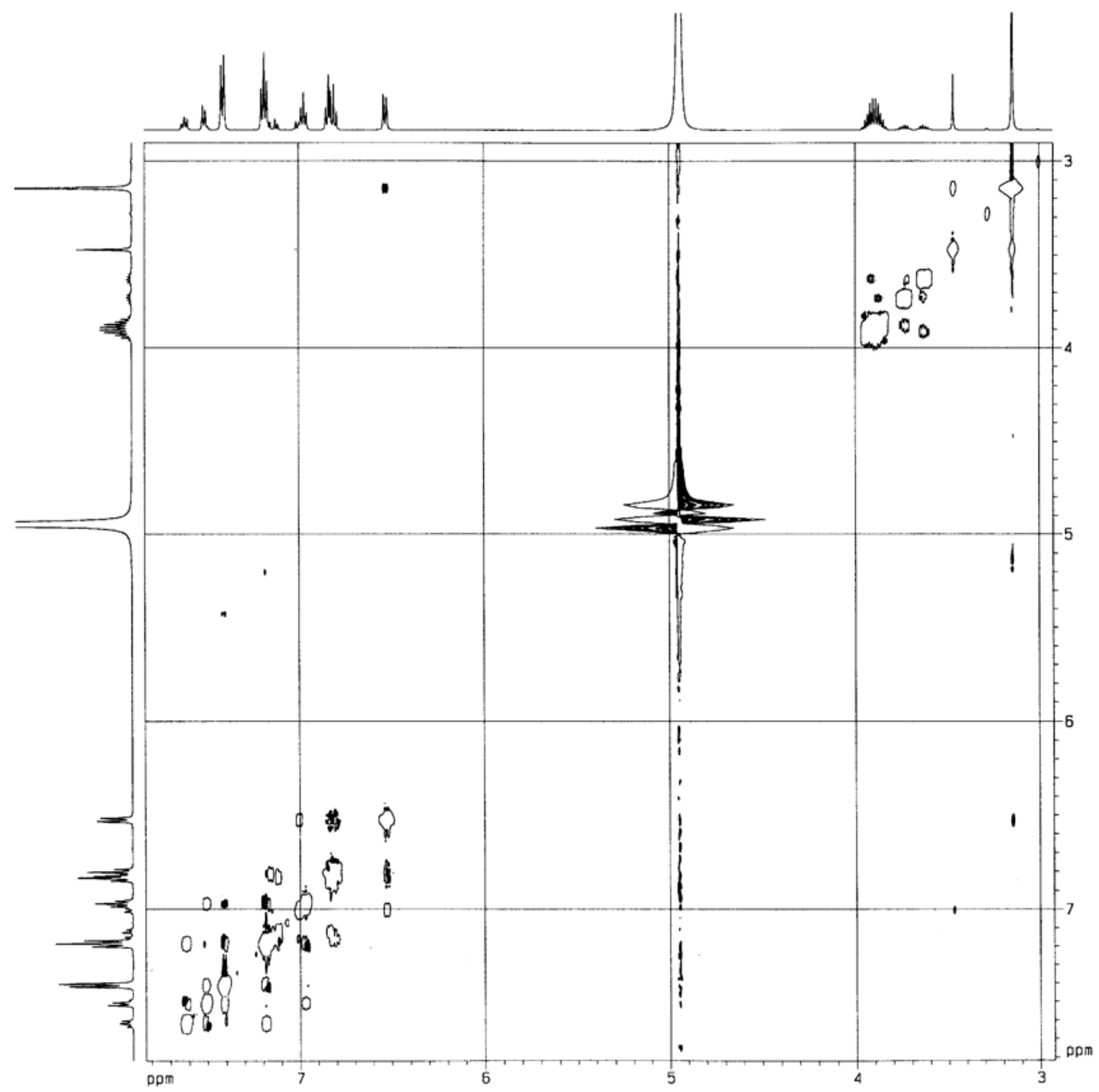

NOESY-spectrum of 2 in $\mathrm{D}_{2} \mathrm{O}+\mathrm{NaOD}$. Signals arising from chemical exchange and remaining COSY correlations are negative and plotted with only one level. NOESY correlations are positive and plotted in several levels. 


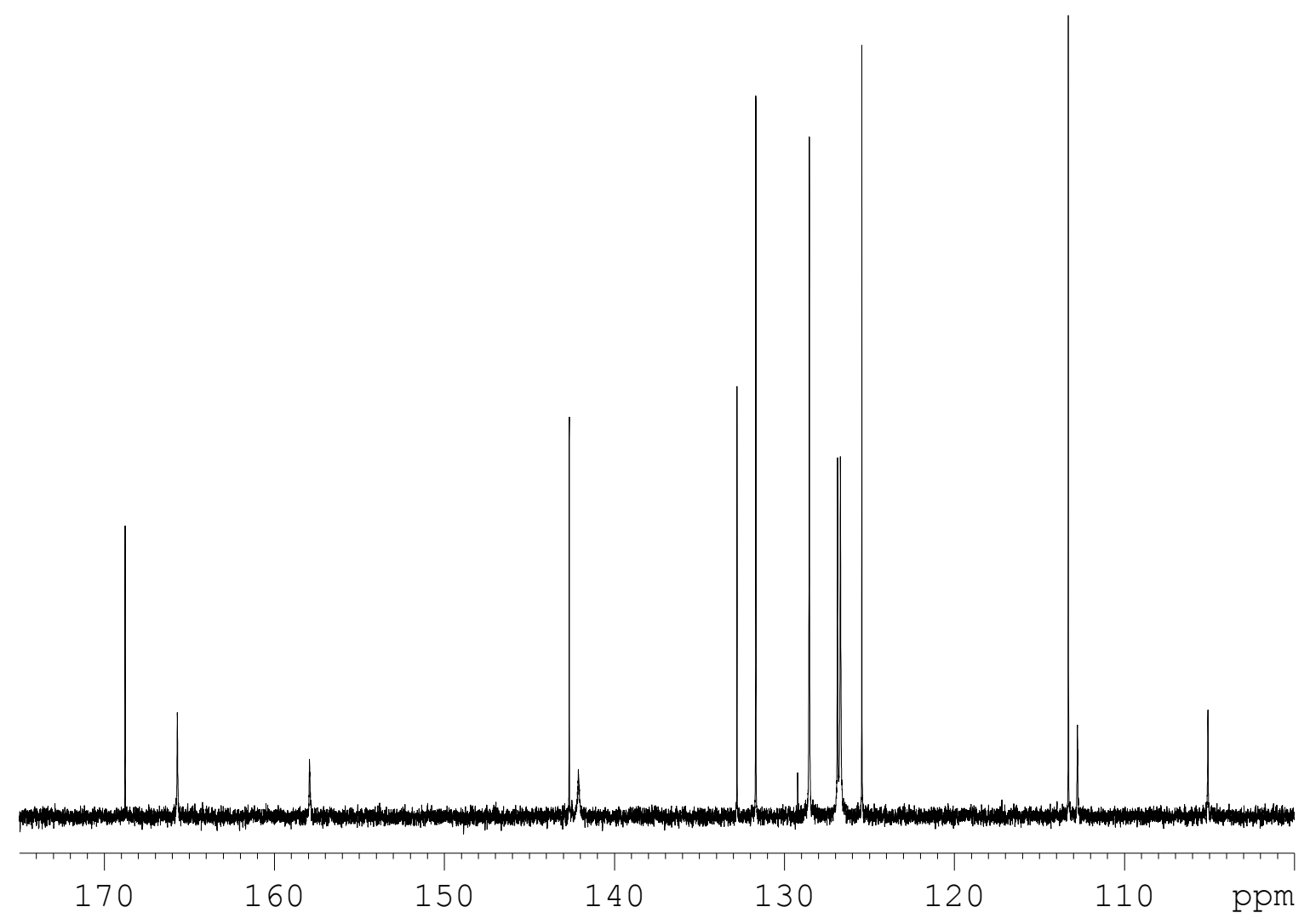

${ }^{13} \mathrm{C}$ NMR spectrum of $\mathbf{2}$ in $\mathrm{CDCl}_{3}$. (Shift presented in Tab. 5).

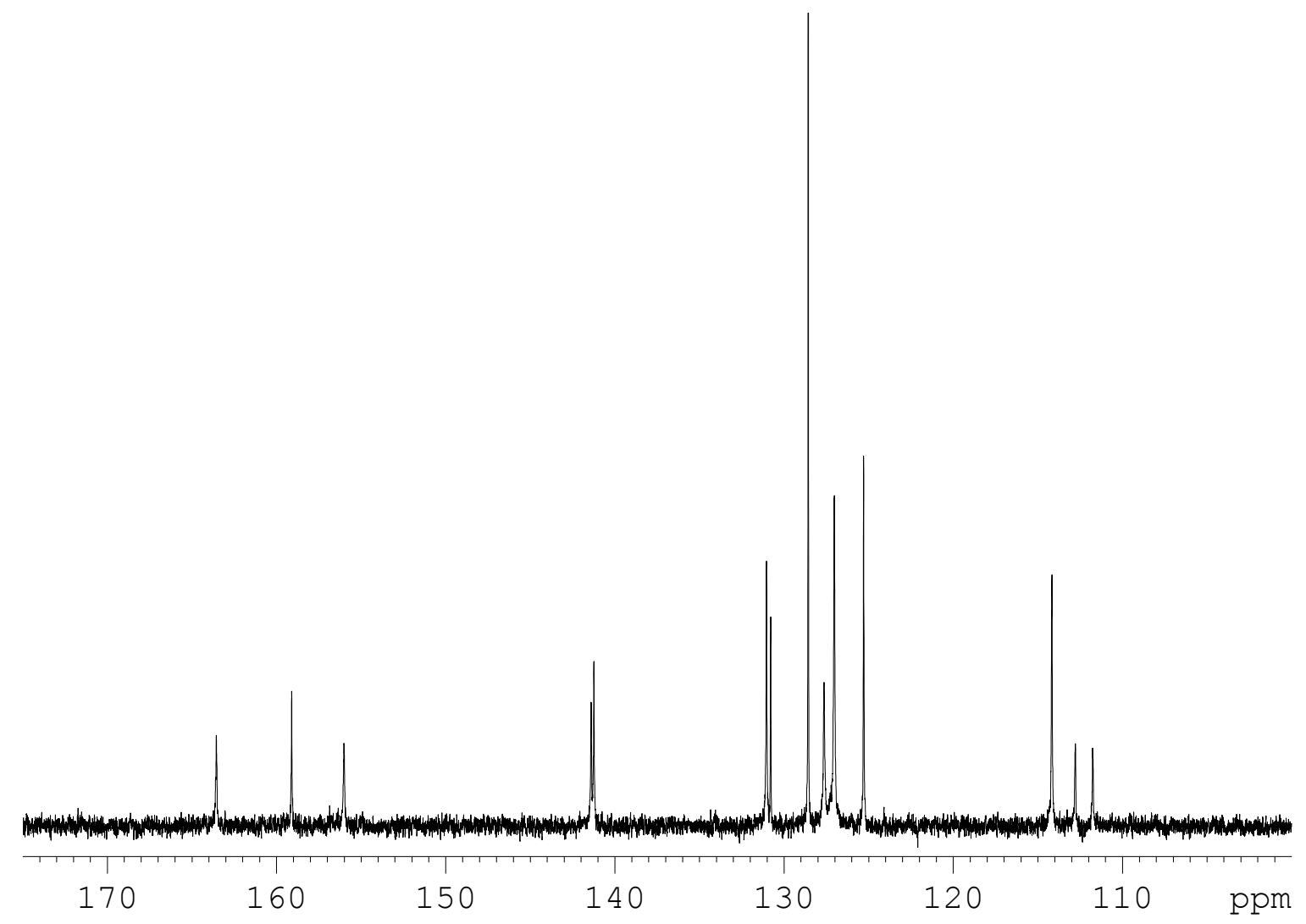

${ }^{13} \mathrm{C}$ NMR spectrum of 2 in $\mathrm{D}_{6}$-DMSO. (Shift presented in Tab. 5). 


\section{Raw data; Impact of the solvent polarity on the reactivity of 2.}

Column 1: Reaction time in days.

Column 2: Concentration of amide $2(\mathrm{mM})$ determined by HPLC.

Column 3: Concentration of sum of formed products 6, 7 and $16(\mathrm{mM})$.

Column 4: Sum of column 2 and $3(\mathrm{mM})$.

Column 5: $\mathrm{Ct}=$ Initial concentration of 2 (Day 0 ) - column 3.

Column 6: $\mathrm{CO} / \mathrm{Ct}=$ Initial concentration of $2($ Day 0$) /$ column 5

Column 7: Natural logaritm of column 6.

\section{Diklormetan}

\begin{tabular}{rrrr} 
Days & \multicolumn{1}{c}{ amid 2 } & \multicolumn{1}{l}{ sum } & \multicolumn{1}{l}{ total } \\
0 & 2.747 & 0 & 2.747 \\
1 & 2.519 & 0.218 & 2.737 \\
2 & 2.348 & 0.394 & 2.742 \\
3 & 2.282 & 0.474 & 2.756
\end{tabular}

\begin{tabular}{rrr} 
Ct * & Co/Ct & In (CO/Ct) \\
2.747 & 1 & 0 \\
2.529 & 1.086200 & 0.082685 \\
2.353 & 1.167446 & 0.154818 \\
2.273 & 1.208535 & 0.189409 \\
\hline
\end{tabular}

Rate constant $=$

\section{Ethanol}

\begin{tabular}{rrrr} 
Days & \multicolumn{1}{c}{ amid 2 } & \multicolumn{1}{l}{ sum } & \multicolumn{1}{c}{ total } \\
0 & 2.853 & 0 & 2.853 \\
1 & 2.811 & 0.057 & 2.868 \\
2 & 2.779 & 0.116 & 2.895 \\
3 & 2.718 & 0.167 & 2.885
\end{tabular}

$\begin{array}{crr}\text { Ct } & \text { Co/Ct } & \text { In (Co/Ct) } \\ 2.853 & 1 & 0 \\ 2.796 & 1.020386 & 0.020181 \\ 2.737 & 1.042382 & 0.041509 \\ 2.686 & 1.062174 & 0.060318\end{array}$

Rate constant $=$

0.020

\section{DMSO}

\begin{tabular}{rrrr} 
Days & \multicolumn{1}{c}{ amid 2 } & \multicolumn{1}{l}{ sum } & \multicolumn{1}{c}{ total } \\
0 & 2.762 & 0 & 2.762 \\
1 & 2.750 & 0.032 & 2.782 \\
2 & 2.692 & 0.058 & 2.750 \\
3 & 2.659 & 0.084 & 2.743
\end{tabular}

$\begin{array}{lr}\text { Rate constant }= & \mathbf{0 . 0 1 0}\end{array}$

$\begin{array}{crr}\text { Ct } & \text { Co/Ct } & \text { In (CO/Ct) } \\ 2.762 & 1 & 0 \\ 2.730 & 1.011722 & 0.011653 \\ 2.704 & 1.021450 & 0.021223 \\ 2.678 & 1.031367 & 0.030885 \\ \text { constant }= & \mathbf{0 . 0 1 0}\end{array}$

\section{$\underline{0.02 \mathrm{M} \mathrm{HCl}}$}

$\begin{array}{rccc}\text { Days } & \text { amid 2 } & \text { sum } & \text { total } \\ 0 & 0.02132 & 0 & 0.02132 \\ 1 & 0.02113 & 0.000068 & 0.02120 \\ 2 & 0.02111 & 0.000105 & 0.02122 \\ 4 & 0.02105 & 0.000162 & 0.02121 \\ 7 & 0.02091 & 0.000333 & 0.02124 \\ 21 & 0.02001 & 0.000891 & 0.02090\end{array}$

\begin{tabular}{crr} 
Ct & \multicolumn{1}{c}{ Co/Ct } & In (Co/Ct) \\
0.021320 & 1 & 0 \\
0.021252 & 1.0032 & 0.003195 \\
0.021215 & 1.004949 & 0.004937 \\
0.021158 & 1.007657 & 0.007628 \\
0.020987 & 1.015867 & 0.015742 \\
0.020429 & 1.043614 & 0.042690
\end{tabular}

Rate constant $=$

0.0020 


\section{Degradation rate for 2 in organic solvents}

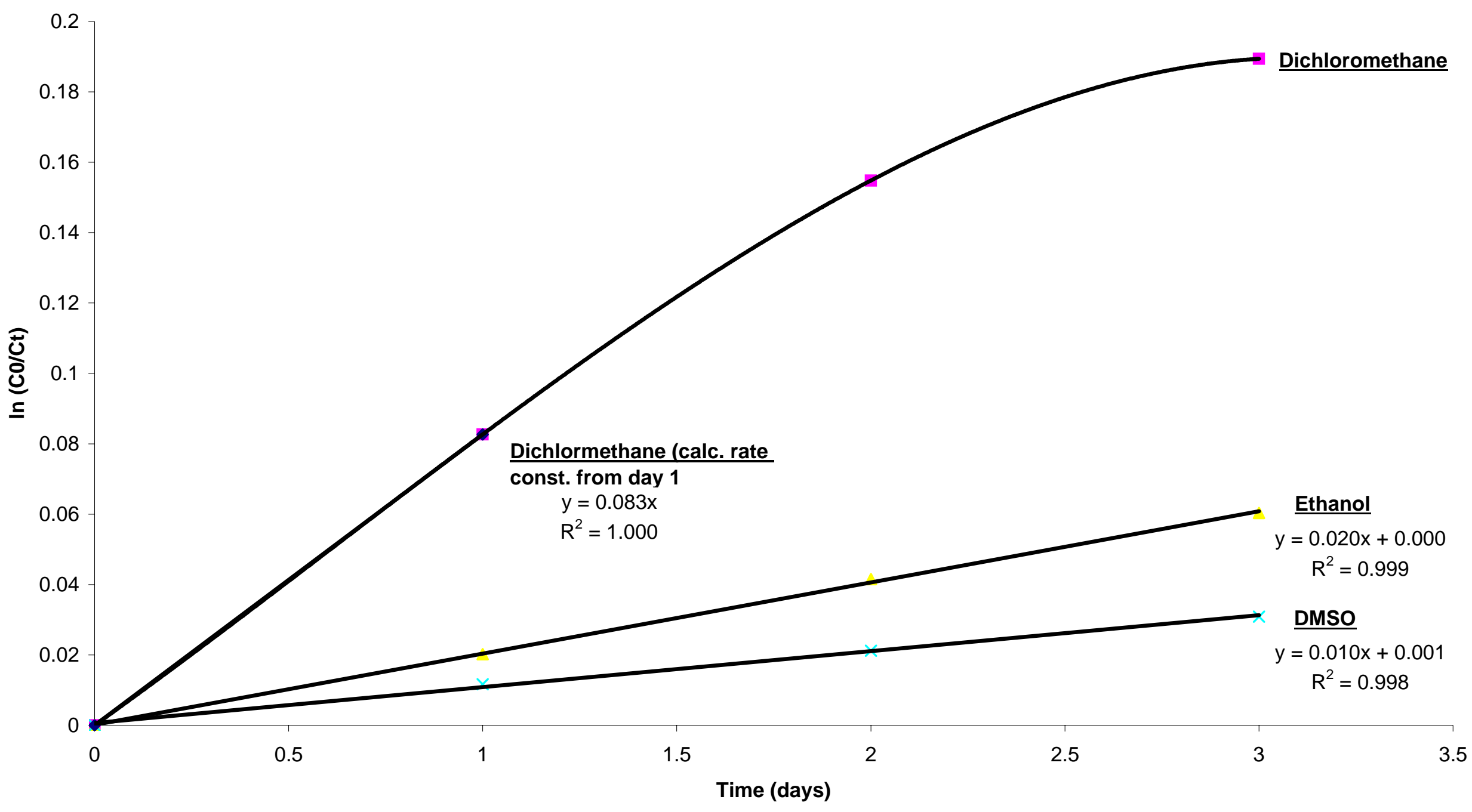




\section{Degradation rate of 2 in $0.02 \mathrm{M} \mathrm{HCl}$}

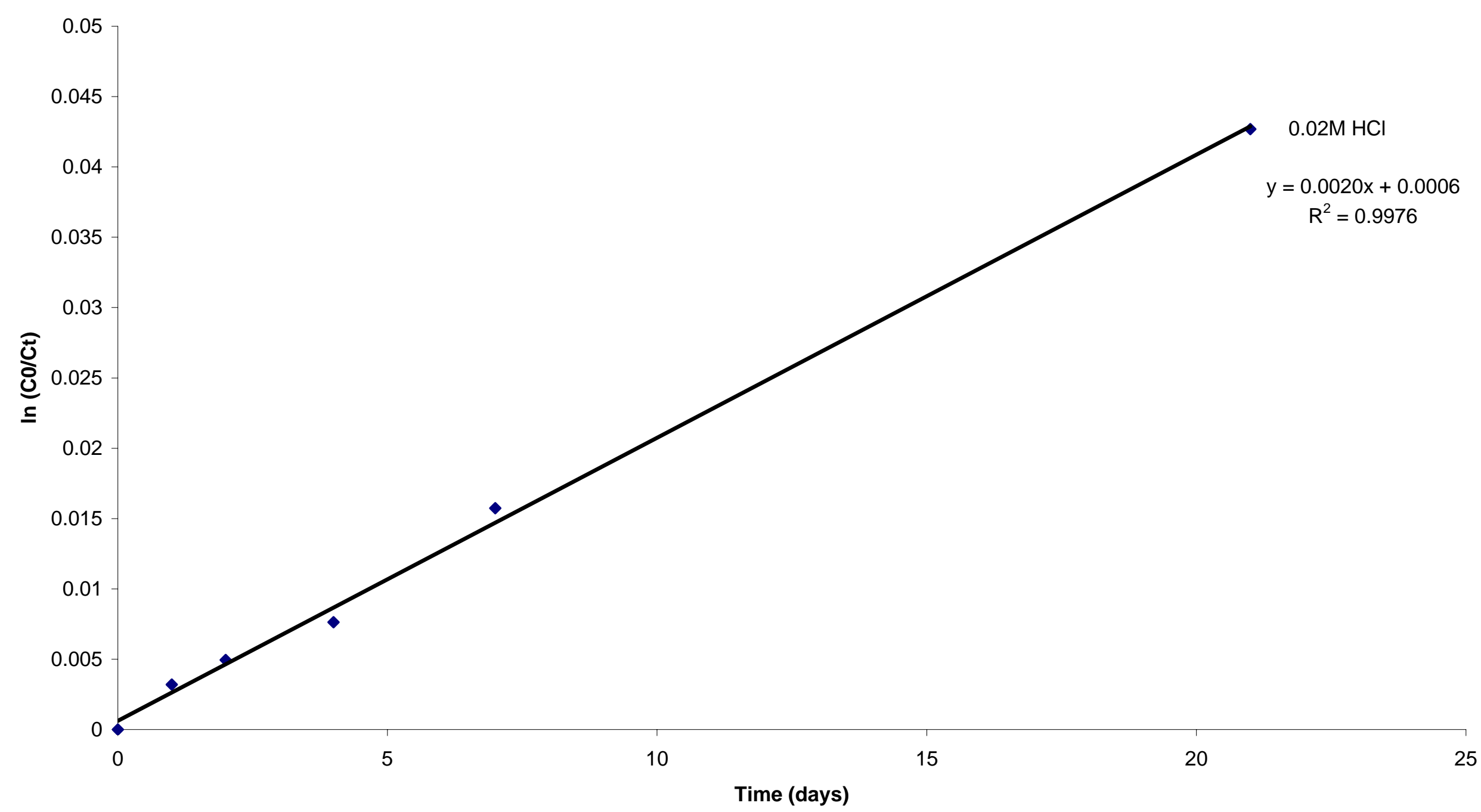




\section{Raw data; kinetic study of the solvolysis of 1 and 2.}

Initial conc. of amide $2(\mathrm{CO})=$

Initial conc. of ester $1(\mathrm{C} 0)=$
$1.103 \mathrm{mM}$

$1.261 \mathrm{mM}$

Column 1: Reaction time in hours.

Column 2: Concentration of amide $2(\mathrm{mM})$ determined by HPLC.

Column 3: Concentration of 7 (mM).

Column 4: Concentration of ester (mM) formed by solvolysis. (n-PrOH-, MeOH-, EtOH- and iso-PrOH-esters are formed in the different exp.)

Column 5: Sum of formed products $=$ sum of column 3 and $4(\mathrm{mM})$.

Column 6: $\mathrm{Cx}=$ Initial concentartion of $\mathbf{2}$ or $\mathbf{1}$ (C0 from above) - concentration of ester (Column 4).

Column 7: $\mathrm{Ct}=$ Initial concentration of $\mathbf{2}$ or $\mathbf{1}$ ( $\mathrm{C} 0$ from above) - concentration of formed products (column 5 ).

Column 8: Natural logaritm of [(C0 from 2 or 1 above)/(Cx from column 6)].

Column 9: Natural logaritm of [(C0 from 2 or 1 above $) /(\mathrm{Ct}$ from column 7$)]$.

Column 10: Natural logaritm of [(C0 from 2 or 1 above $) /(\mathrm{COt}$ from column 2)].

\section{Amide 2 and $1.6 \% \mathrm{n}-\mathrm{PrOH}$.}

\begin{tabular}{|c|c|c|c|c|c|c|c|c|c|}
\hline Hours & $2(\mathrm{COt})$ & 7 & ester & sum & Cx & $\mathbf{C t}$ & In $C 0 / C x$ & In $\mathrm{CO} / \mathrm{Ct}$ & In Co/COt \\
\hline 0 & 1.1141 & 0 & 0 & 0 & 1.1030 & 1.1030 & 0 & 0 & -0.010013 \\
\hline 1 & 0.8547 & 0.0152 & 0.1998 & 0.2150 & 0.9032 & 0.8880 & 0.199845 & 0.216817 & 0.255038 \\
\hline 2 & 0.7014 & 0.0275 & 0.3724 & 0.3999 & 0.7306 & 0.7031 & 0.411923 & 0.450290 & 0.452711 \\
\hline 3 & 0.5602 & 0.0828 & 0.4615 & 0.5443 & 0.6415 & 0.5587 & 0.541980 & 0.680176 & 0.677495 \\
\hline 4 & 0.4530 & 0.0457 & 0.6221 & 0.6678 & 0.4809 & 0.4352 & 0.830130 & 0.929983 & 0.889897 \\
\hline \multicolumn{6}{|c|}{ Mean: $91 \%$ of the products is ester. } & Rate constant $=$ & 0.200 & 0.232 & 0.222 \\
\hline Hours & $2(\mathrm{COt})$ & 7 & ester & sum & Cx & Ct & In $\mathrm{CO} / \mathrm{Cx}$ & In CO/Ct & In $\mathrm{CO} / \mathrm{COt}$ \\
\hline 0 & 1.1113 & 0 & 0 & 0 & 1.1030 & 1.1030 & 0 & 0 & -0.007497 \\
\hline 1 & 0.8669 & 0.0597 & 0.1582 & 0.2179 & 0.9448 & 0.8851 & 0.154816 & 0.220088 & 0.240865 \\
\hline 2 & 0.7027 & 0.1119 & 0.2867 & 0.3986 & 0.8163 & 0.7044 & 0.301007 & 0.448443 & 0.450859 \\
\hline 3 & 0.5667 & 0.1513 & 0.3922 & 0.5435 & 0.7108 & 0.5595 & 0.439398 & 0.678745 & 0.665959 \\
\hline 4 & 0.4675 & 0.2078 & 0.4495 & 0.6573 & 0.6535 & 0.4457 & 0.523446 & 0.906143 & 0.858390 \\
\hline \multicolumn{6}{|c|}{ Mean $71 \%$ of the products is ester. } & Rate constant $=$ & 0.133 & 0.227 & 0.216 \\
\hline
\end{tabular}


Amide 2 and $0.32 \%$ iso-PrOH.

\begin{tabular}{ccrrr} 
Hours & $\mathbf{2}(\mathbf{C O t})$ & \multicolumn{1}{c}{ ester } & \multicolumn{1}{c}{ sum } \\
0 & 1.1174 & 0 & 0 & 0 \\
1 & 0.8795 & 0.0881 & 0.1322 & 0.2203 \\
2 & 0.7157 & 0.1569 & 0.2399 & 0.3968 \\
3 & 0.5550 & 0.2080 & 0.3161 & 0.5241 \\
4 & 0.4661 & 0.2580 & 0.3875 & 0.6455 \\
Mean: $60 \%$ & of the products is ester
\end{tabular}

$\begin{array}{ll}\mathbf{C x} & \mathbf{C t} \\ 1.1030 & 1.1030 \\ 0.9708 & 0.8827 \\ 0.8631 & 0.7062 \\ 0.7869 & 0.5789 \\ 0.7155 & 0.4575\end{array}$

$\begin{array}{rr}\text { In } \mathbf{C O} / \mathbf{C x} & \ln \mathbf{C O} / \mathbf{C t} \\ 0 & 0 \\ 0.127669 & 0.222804 \\ 0.245258 & 0.445891 \\ 0.337688 & 0.644659 \\ 0.432807 & 0.880012 \\ \mathbf{0 . 1 0 8} & \mathbf{0 . 2 1 8}\end{array}$

In $\mathrm{CO} / \mathrm{COt}$

$-0.012971$

0.226435

0.432528

0.686821

0.861389

Rate constant $=\quad 0.108 \quad 0.218 \quad 0.221$

\section{Ester 1 and $1.6 \% \mathrm{n}-\mathrm{PrOH}$.}

\begin{tabular}{ccrrr} 
Hours & $\mathbf{1}$ (C)t) & $\mathbf{7}$ & \multicolumn{1}{c}{ ester } & \multicolumn{1}{c}{ sum } \\
0 & 1.2954 & 0 & 0 & 0 \\
2 & 1.2330 & 0.0008 & 0.0077 & 0.0085 \\
4 & 1.2313 & 0.0011 & 0.0155 & 0.0166 \\
6 & 1.2210 & 0.0018 & 0.0231 & 0.0249 \\
24 & 1.1583 & 0.0113 & 0.0881 & 0.0994 \\
\multicolumn{5}{c}{ Mean: $91 \%$ of the products is ester. }
\end{tabular}

Eater 1 and $0.064 \%$ n-PrOH.

\begin{tabular}{cccrrr} 
Hours & $\mathbf{1}(\mathbf{C} \mathbf{0 t})$ & $\mathbf{7}$ & \multicolumn{1}{c}{ ester } & sum \\
0 & 1.2218 & 0 & 0 & 0 \\
2 & 1.2388 & 0.0058 & 0.0016 & 0.0074 \\
4 & 1.2328 & 0.0111 & 0.0038 & 0.0149 \\
6 & 1.2200 & 0.0173 & 0.0060 & 0.0233 \\
24 & 1.1692 & 0.0649 & 0.0235 & 0.0884 \\
& \multicolumn{4}{c}{ Mean: $25 \%$ of the products is ester. }
\end{tabular}

Cx

1.2610

1.2533

1.2455

1.2379

1.1729

\section{Ct}

1.2610

1.2525

1.2444

1.2361

1.1616

Rate constant $=$
In $\mathrm{Co} / \mathrm{Cx}$ In $\mathrm{CO} / \mathrm{Ct}$
0
$\begin{array}{lll}0.006125 & 0.006764\end{array}$
$0.012368 \quad 0.013252$
$0.018489 \quad 0.019944$
$0.072426 \quad 0.082107$
0.003010 .00343

In $\mathrm{CO} / \mathrm{COt}$

$-0.026914$

0.022455

0.023835

0.032235

0.084952

0.00373

$\begin{array}{llrr}\mathbf{C x} & \mathbf{C t} & \mathbf{I n} \mathbf{C 0 / C \mathbf { x }} & \mathbf{l n} \mathbf{C 0 / C t} \\ 1.2610 & 1.2610 & 0 & 0 \\ 1.2594 & 1.2536 & 0.001270 & 0.005886 \\ 1.2572 & 1.2461 & 0.003018 & 0.011886 \\ 1.2550 & 1.2377 & 0.004769 & 0.018650 \\ 1.2375 & 1.1726 & 0.018812 & 0.072682 \\ & \text { Rate constant }= & \mathbf{0 . 0 0 0 7 9} & \mathbf{0 . 0 0 3 0 3}\end{array}$

In $\mathrm{CO} / \mathrm{COt}$

0.031580

0.017762

0.022617

0.033054

0.075585

0.00225 
Amide 2 and $0.064 \% \mathrm{n}$-PrOH.

\begin{tabular}{ccrrrr} 
Hours & $\mathbf{2}$ (C)t) & \multicolumn{7}{c}{ ester } & \multicolumn{1}{c}{ sum } \\
& 0 & 1.0739 & 0 & 0 & 0 \\
1 & 0.8750 & 0.1483 & 0.0728 & 0.2211 \\
2 & 0.7005 & 0.2638 & 0.1279 & 0.3917 \\
3 & 0.5761 & 0.3697 & 0.1733 & 0.5430 \\
4 & 0.4756 & 0.4488 & 0.2069 & 0.6557 \\
Mean: $32 \%$ of the products is ester.
\end{tabular}

$\begin{array}{llrrr}\mathbf{C x} & \mathbf{C t} & \text { In } \mathbf{C 0 / C x} & \text { In } \mathbf{C 0} / \mathbf{C t} & \text { In } \mathbf{C o / C 0 t} \\ 1.1030 & 1.1030 & 0 & 0 & 0.026737 \\ 1.0302 & 0.8819 & 0.068281 & 0.223710 & 0.231565 \\ 0.9751 & 0.7113 & 0.123249 & 0.438695 & 0.453995 \\ 0.9297 & 0.5600 & 0.170927 & 0.677852 & 0.649508 \\ 0.8961 & 0.4473 & 0.207737 & 0.902560 & 0.841212 \\ & \text { Rate constant }= & \mathbf{0 . 0 5 2} & \mathbf{0 . 2 2 6} & \mathbf{0 . 2 0 5}\end{array}$

\section{Amide 2 and $0.32 \% \mathrm{MeOH}$.}

\begin{tabular}{ccrrr} 
Hours & $\mathbf{2}$ (C0t) & $\mathbf{7}$ & \multicolumn{1}{c}{ ester } & sum \\
0 & 1.1516 & 0 & 0 & 0 \\
1 & 0.8753 & 0.0537 & 0.1634 & 0.2171 \\
2 & 0.6984 & 0.0603 & 0.3243 & 0.3846 \\
3 & 0.5611 & 0.0821 & 0.4409 & 0.5230 \\
4 & 0.4757 & 0.1024 & 0.5452 & 0.6476 \\
Mean: $82 \%$ of the products is ester.
\end{tabular}

$\begin{array}{ll}\mathbf{C x} & \mathbf{C t} \\ 1.1030 & 1.1030 \\ 0.9396 & 0.8859 \\ 0.7787 & 0.7184 \\ 0.6621 & 0.5800 \\ 0.5578 & 0.4554 \\ & \text { Rate }\end{array}$

Ct

0.8859

0.7184

0.4554

Rate constant =
In $\mathrm{CO} / \mathrm{Cx}$ In $\mathrm{CO} / \mathrm{Ct}$ 0
$0.160335 \quad 0.219185$
$0.348163 \quad 0.428763$
$0.510372 \quad 0.642761$
$0.681789 \quad 0.884613$
$0.171 \quad 0.219$

In $\mathrm{Co} / \mathrm{COt}$

$-0.043119$

0.231222

0.456997

0.675890

0.841002

0.221

\section{Amide 2 and $0.32 \% \mathrm{EtOH}$.}

\begin{tabular}{ccrrr} 
Hours & $\mathbf{2}$ (C)t) & $\mathbf{7}$ & ester & sum \\
0 & 1.0870 & 0 & 0 & 0 \\
1 & 0.8803 & 0.1019 & 0.1215 & 0.2234 \\
2 & 0.7068 & 0.1303 & 0.2616 & 0.3919 \\
3 & 0.5806 & 0.1389 & 0.3981 & 0.5370 \\
4 & 0.4701 & 0.1799 & 0.4685 & 0.6484 \\
& \multicolumn{4}{c}{ Mean: $66 \%$ of the products is ester. }
\end{tabular}

Cx Ct

$1.1030 \quad 1.1030$

$0.9815 \quad 0.8796$

$0.8414 \quad 0.7111$

$0.7049 \quad 0.5660$

0.6345

$\begin{array}{rr}\text { In } \mathbf{C 0 / C x} & \text { In } \mathbf{C O} / \mathbf{C t} \\ 0 & 0 \\ 0.116707 & 0.226322 \\ 0.270722 & 0.438976 \\ 0.447733 & 0.667195 \\ 0.552952 & 0.886371 \\ \mathbf{0 . 1 4 4} & \mathbf{0 . 2 2 1}\end{array}$

In Co/COt

0.014612

0.225526

0.445041

0.641727

0.852844

0.209 


\section{Amide 2 and $1.6 \%$ n-PrOH}

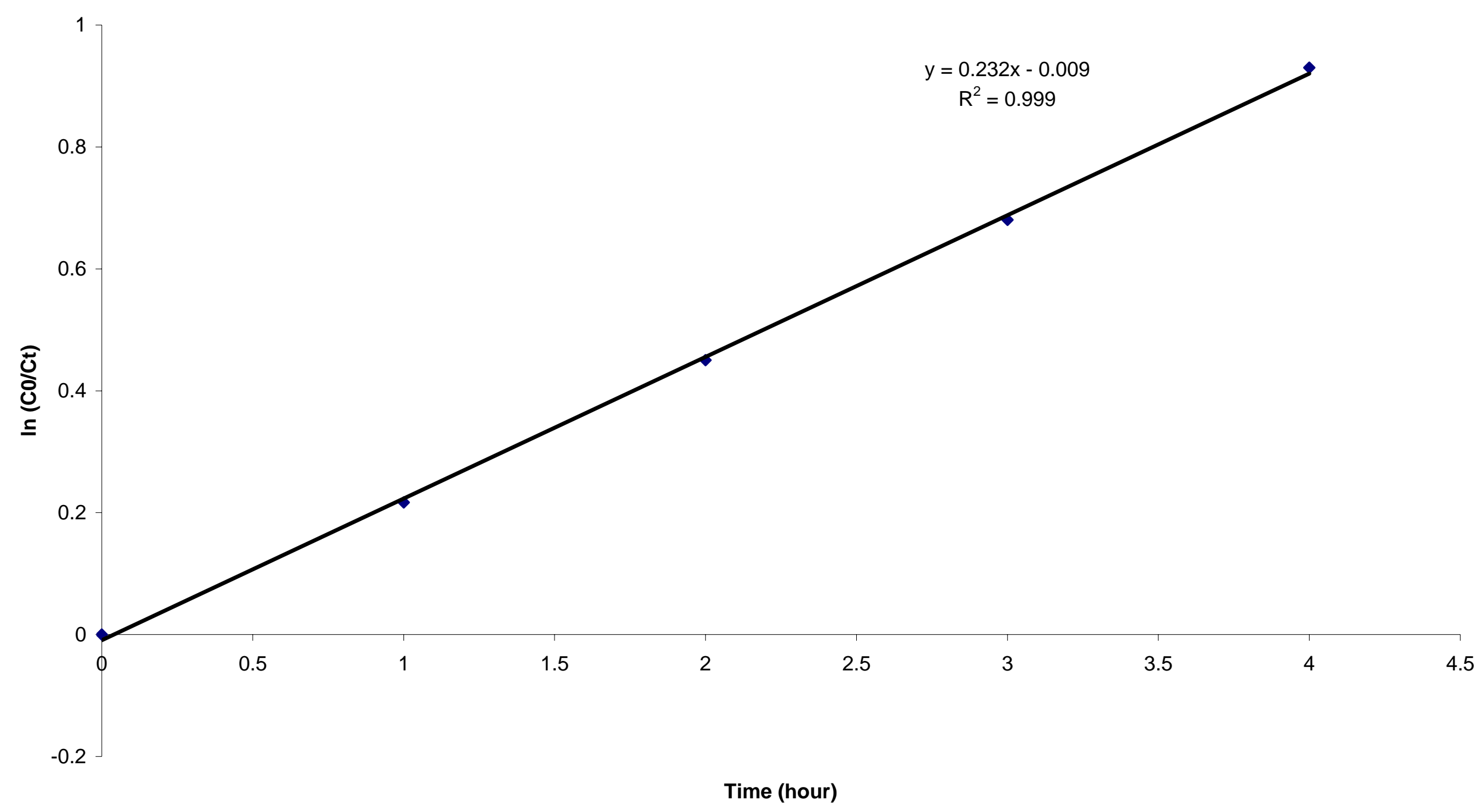




\section{Amide 2 and $1.6 \%$ n-PrOH}

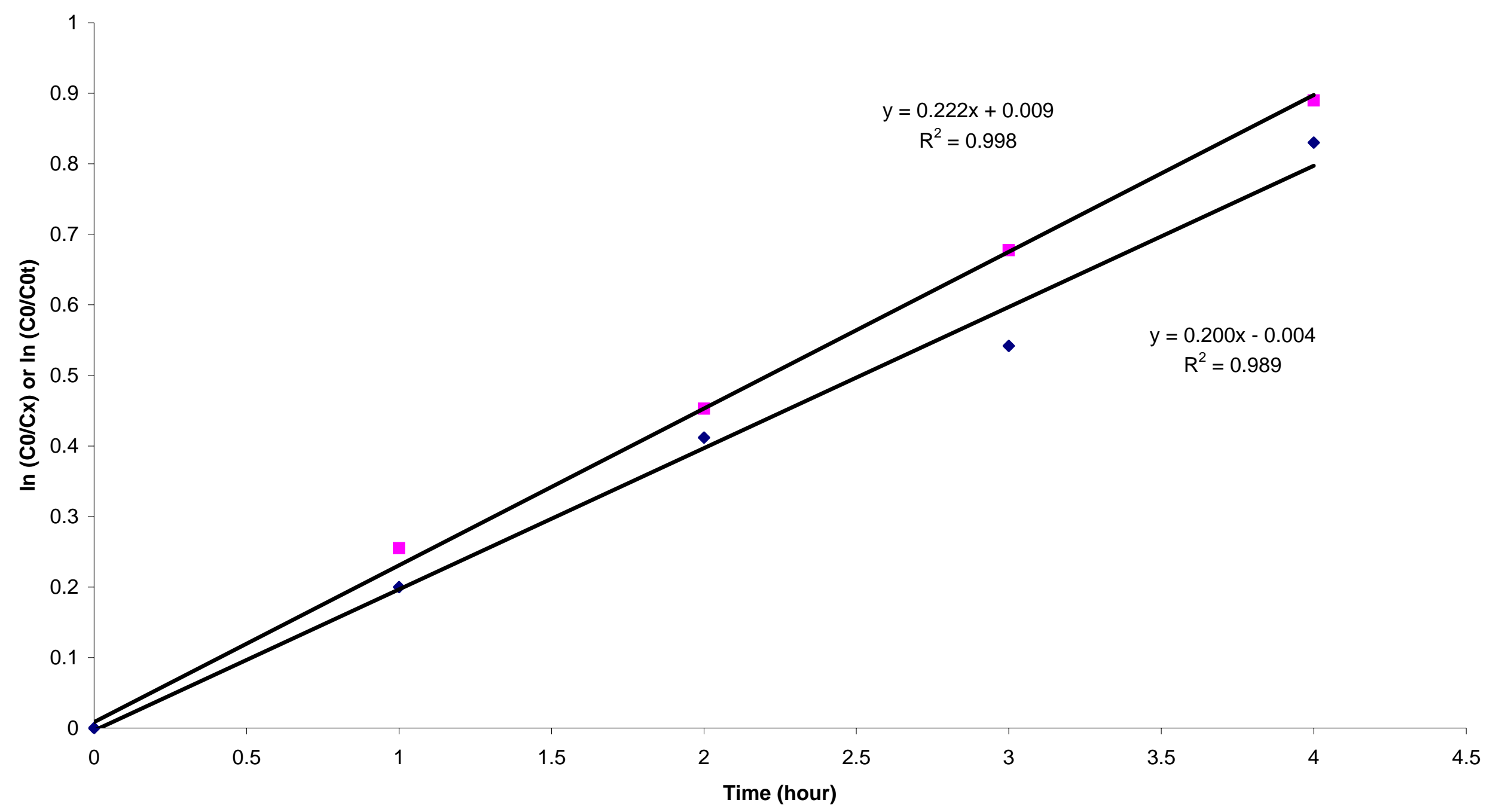




\section{Amide 2 and $0.32 \% \mathrm{n}-\mathrm{PrOH}$}

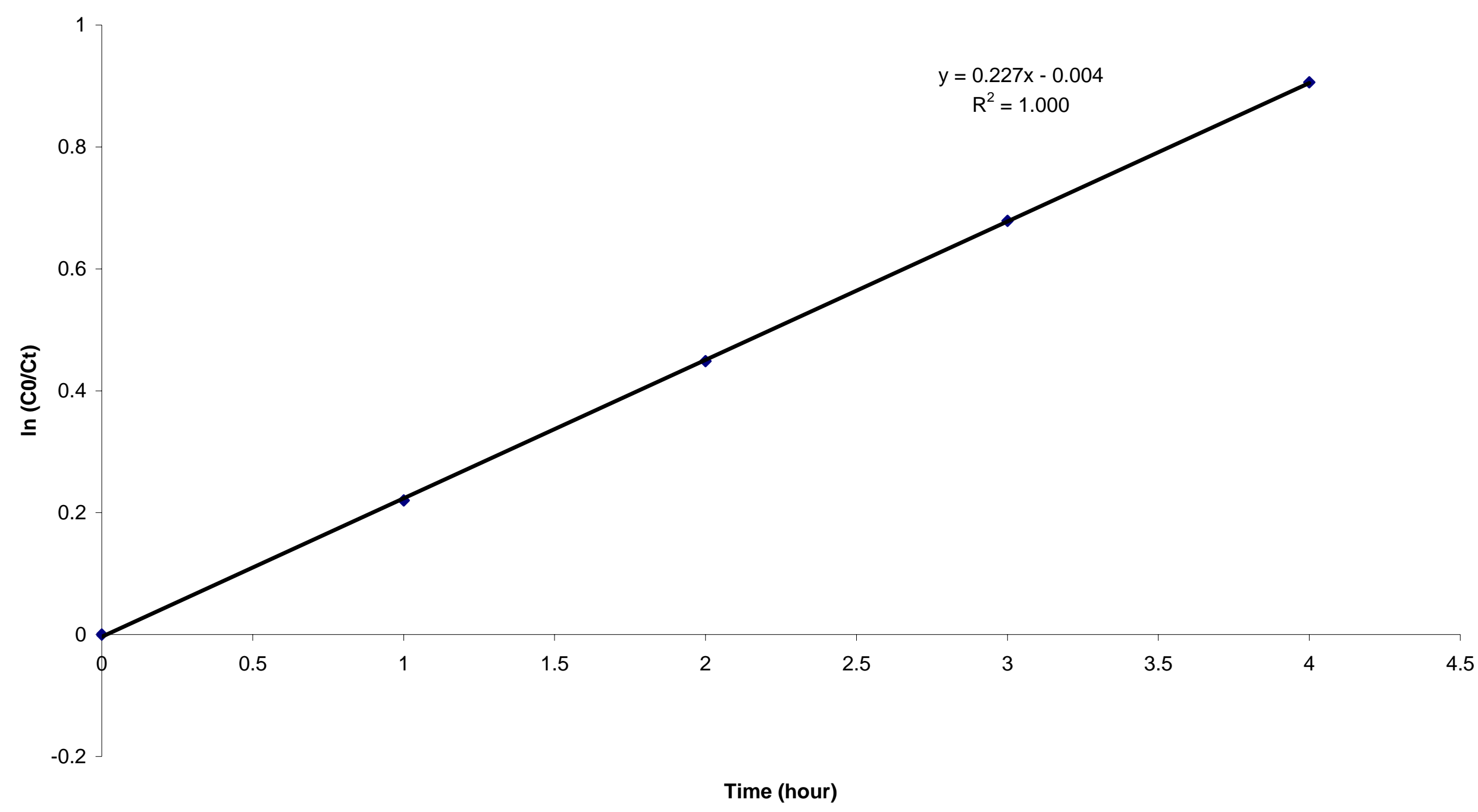




\section{Amide 2 and $0.32 \%$ n-PrOH}

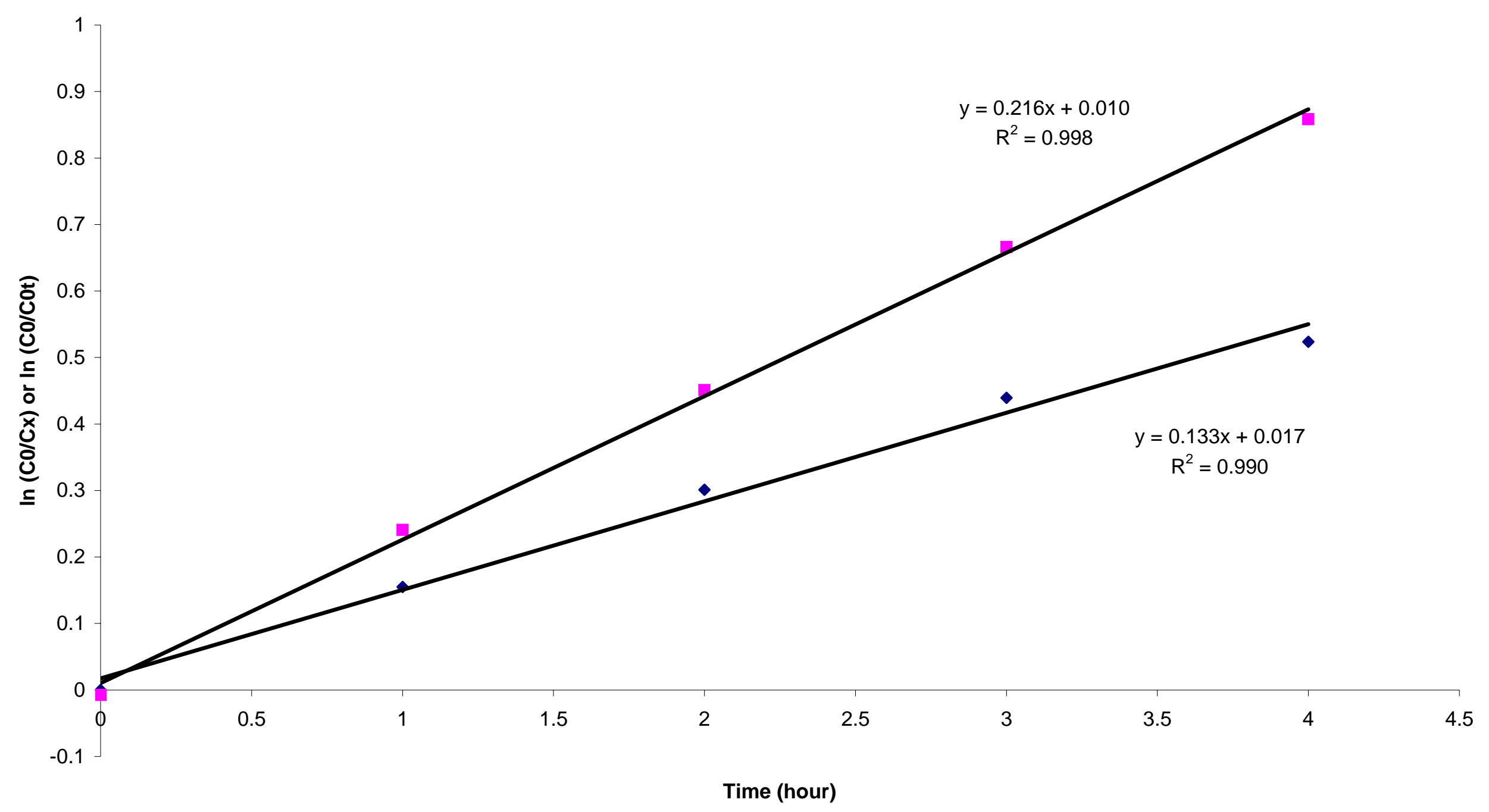


Amide 2 and $0.064 \% \mathrm{n}-\mathrm{PrOH}$

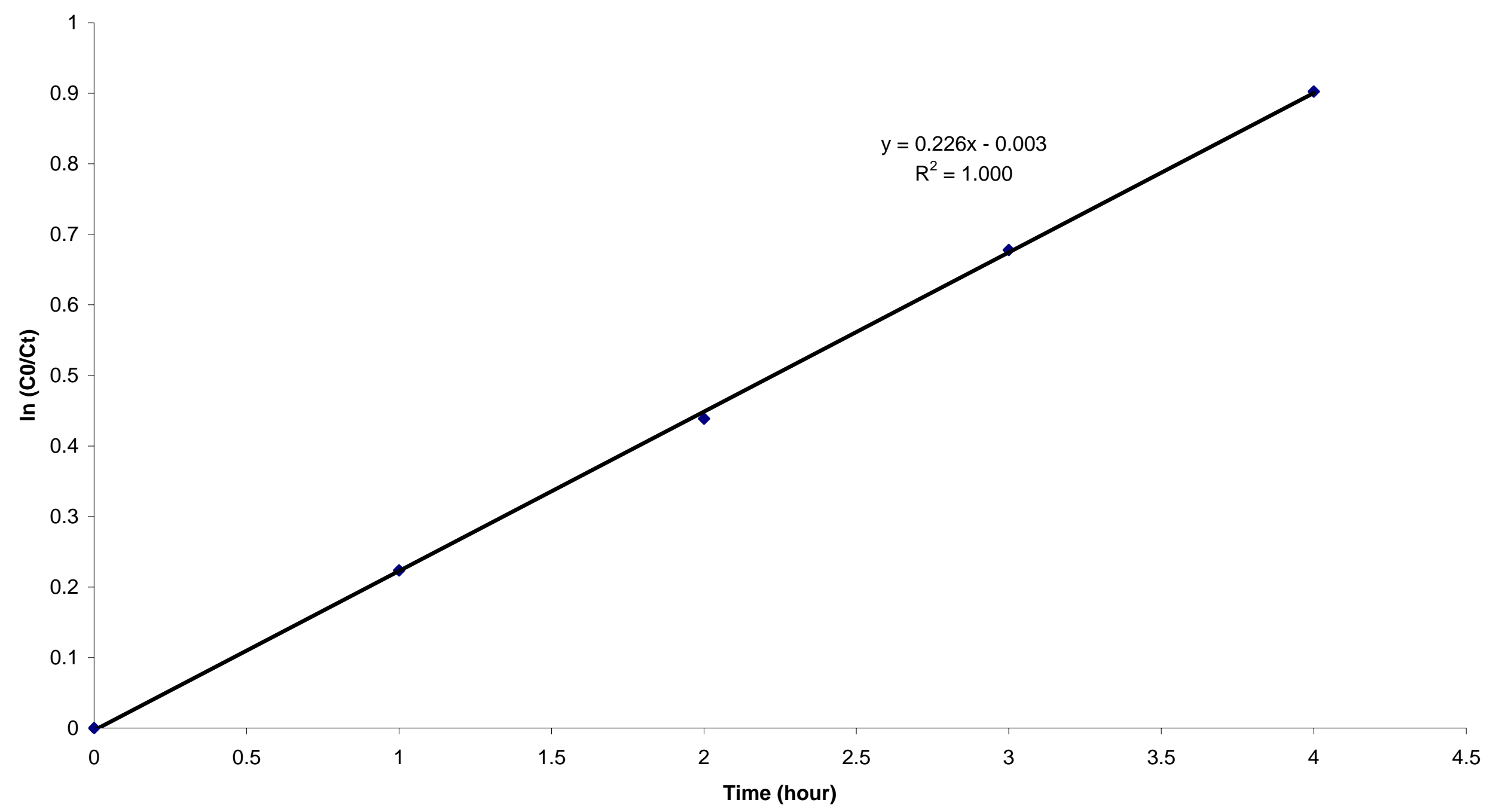


Amide 2 and $0.064 \%$ n-PrOH

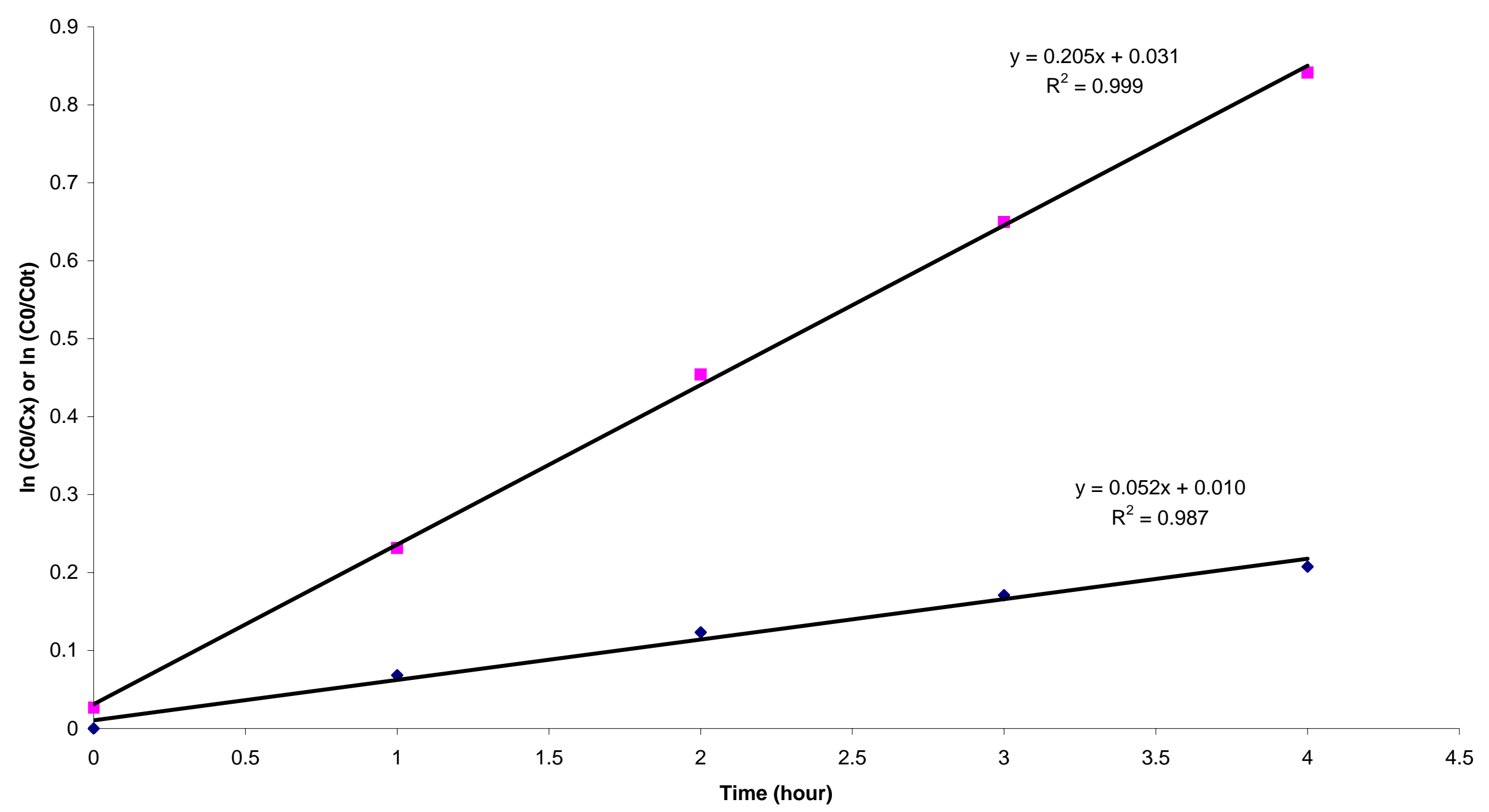




\section{Amide 2 and $0.32 \% \mathrm{MeOH}$}

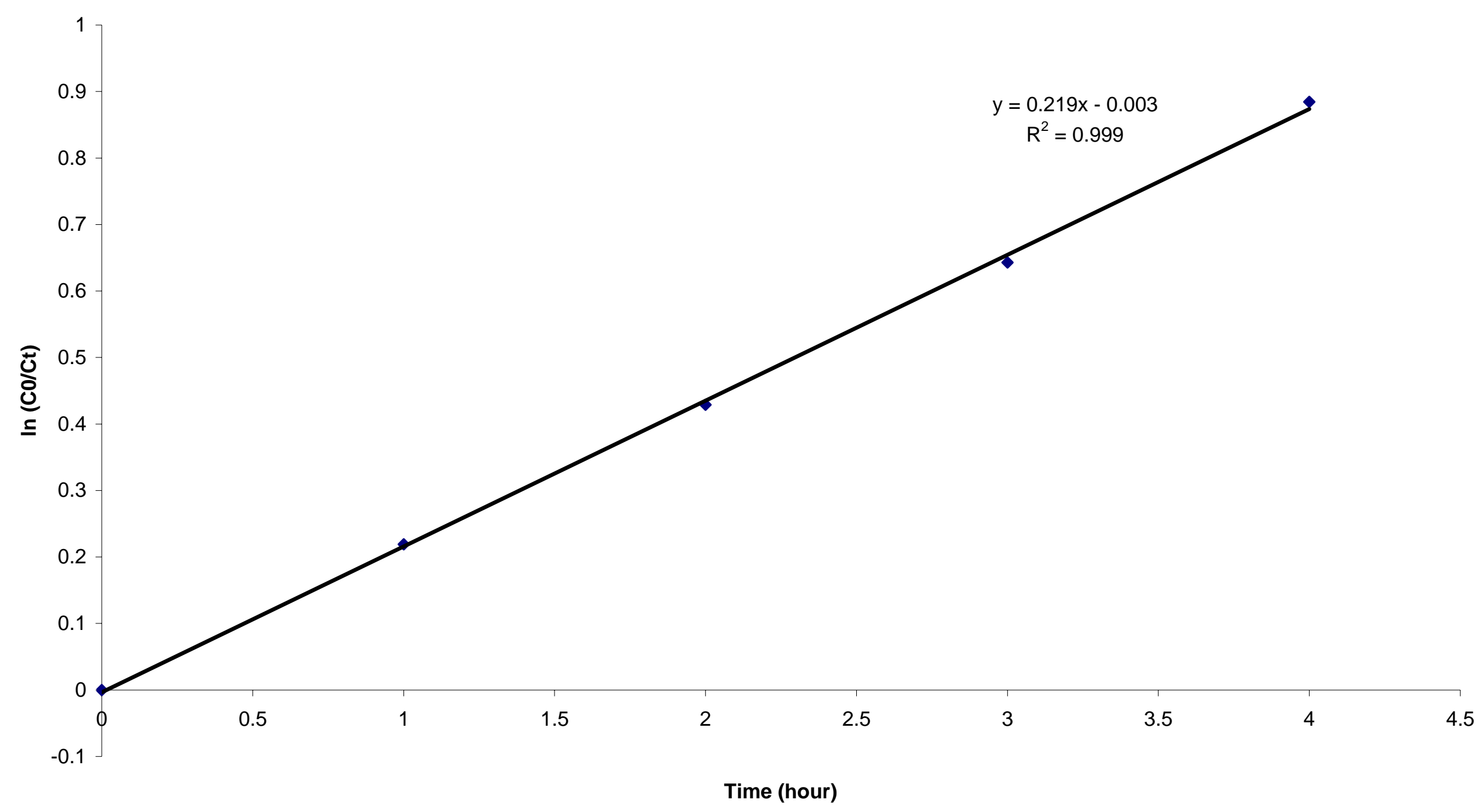




\section{Amide 2 and $0.32 \% \mathrm{MeOH}$}

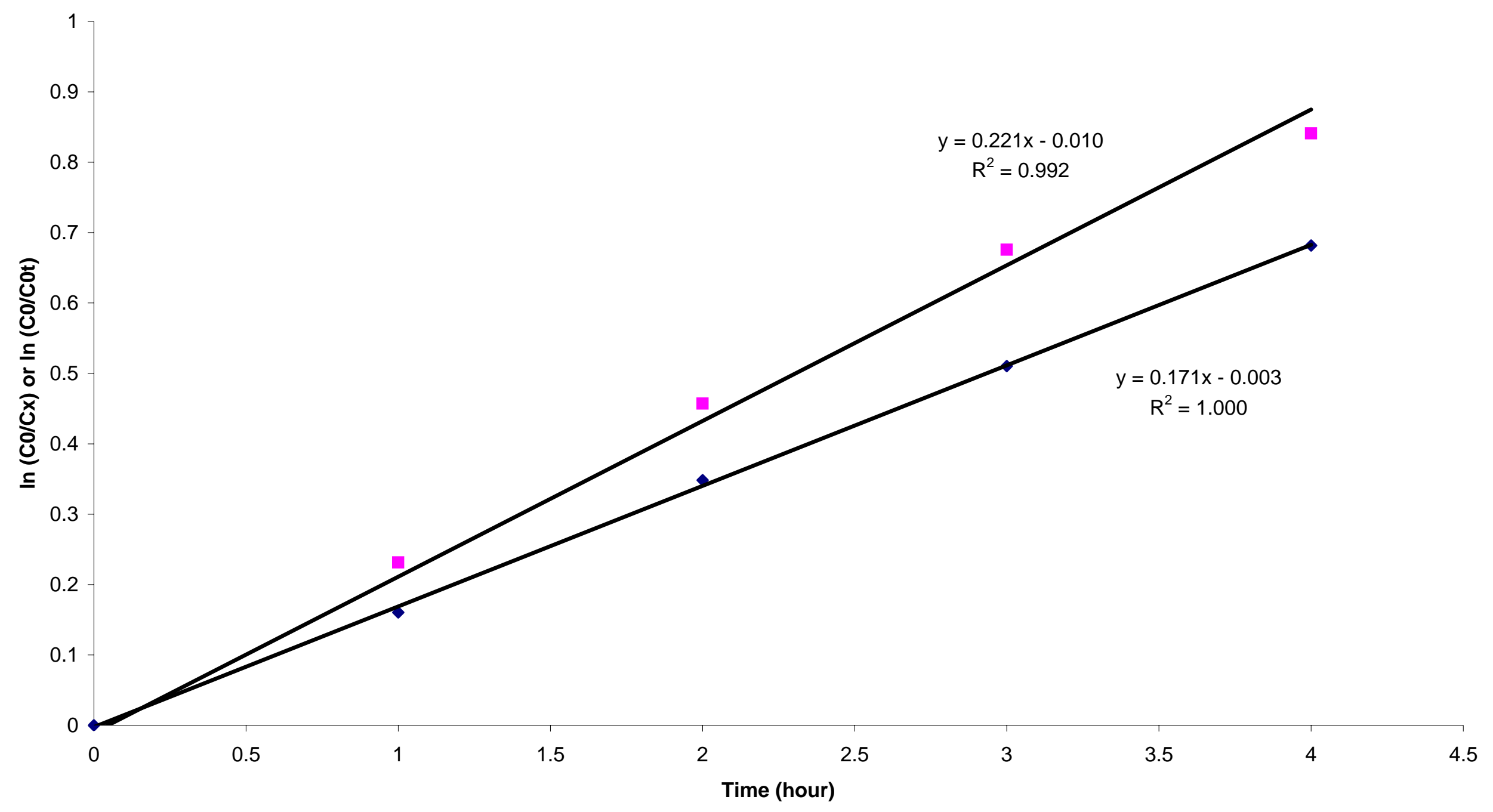


Amide 2 and $0.32 \%$ EtOH

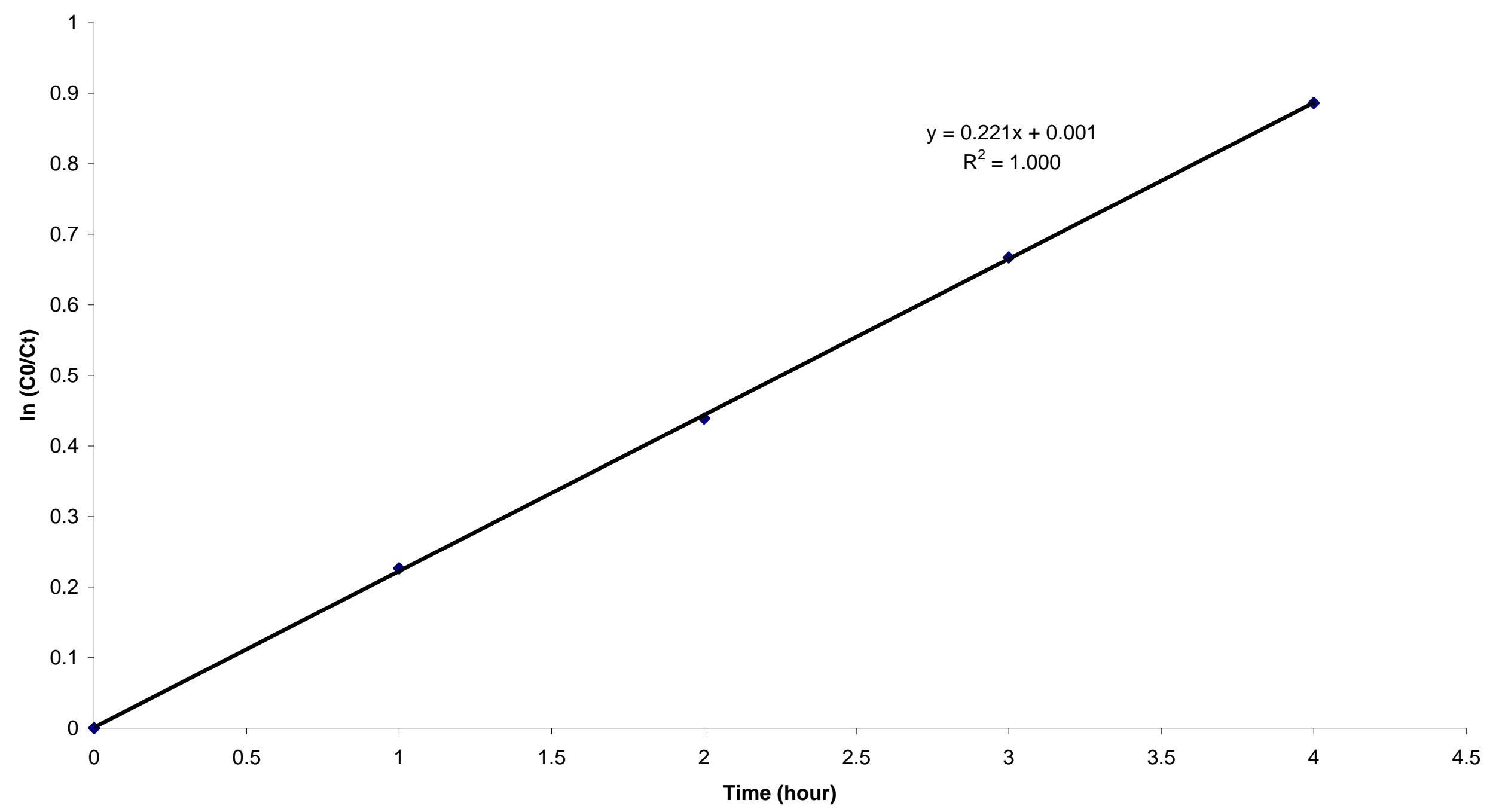


Amide 2 and $0.32 \%$ EtOH

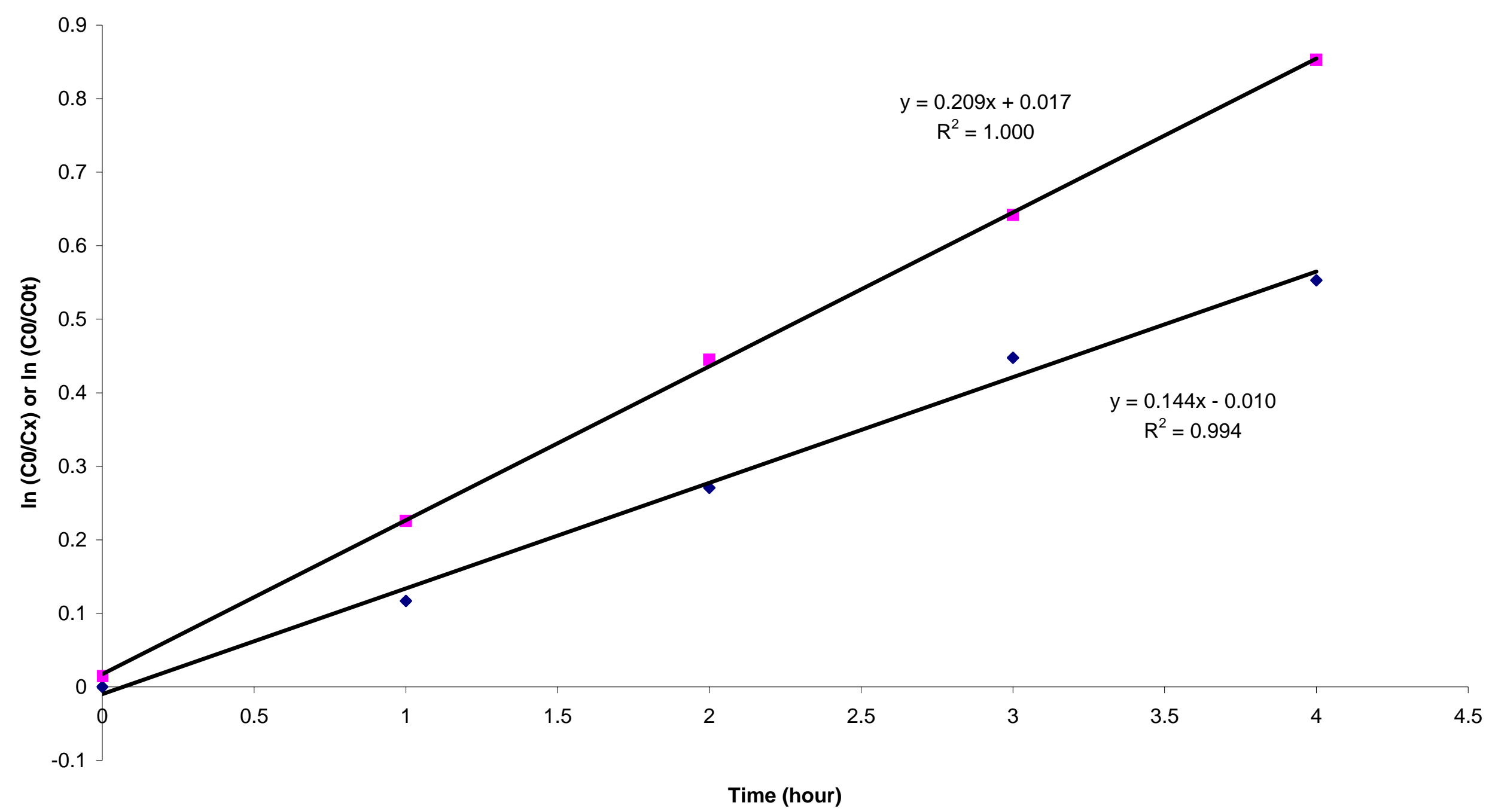




\section{Amide 2 and $0.32 \%$ iso-PrOH}

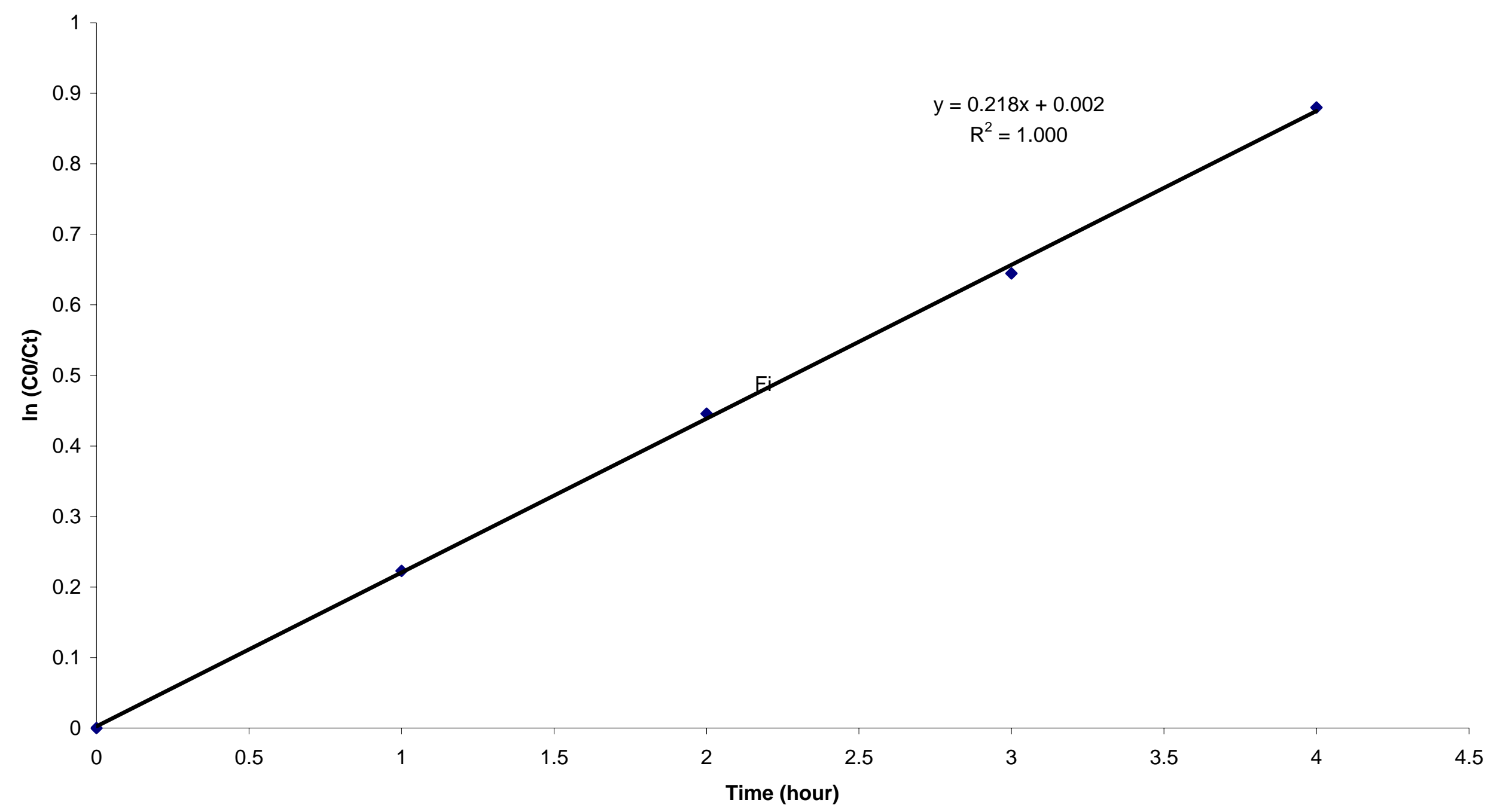




\section{Amide 2 and $0.32 \%$ iso-PrOH}

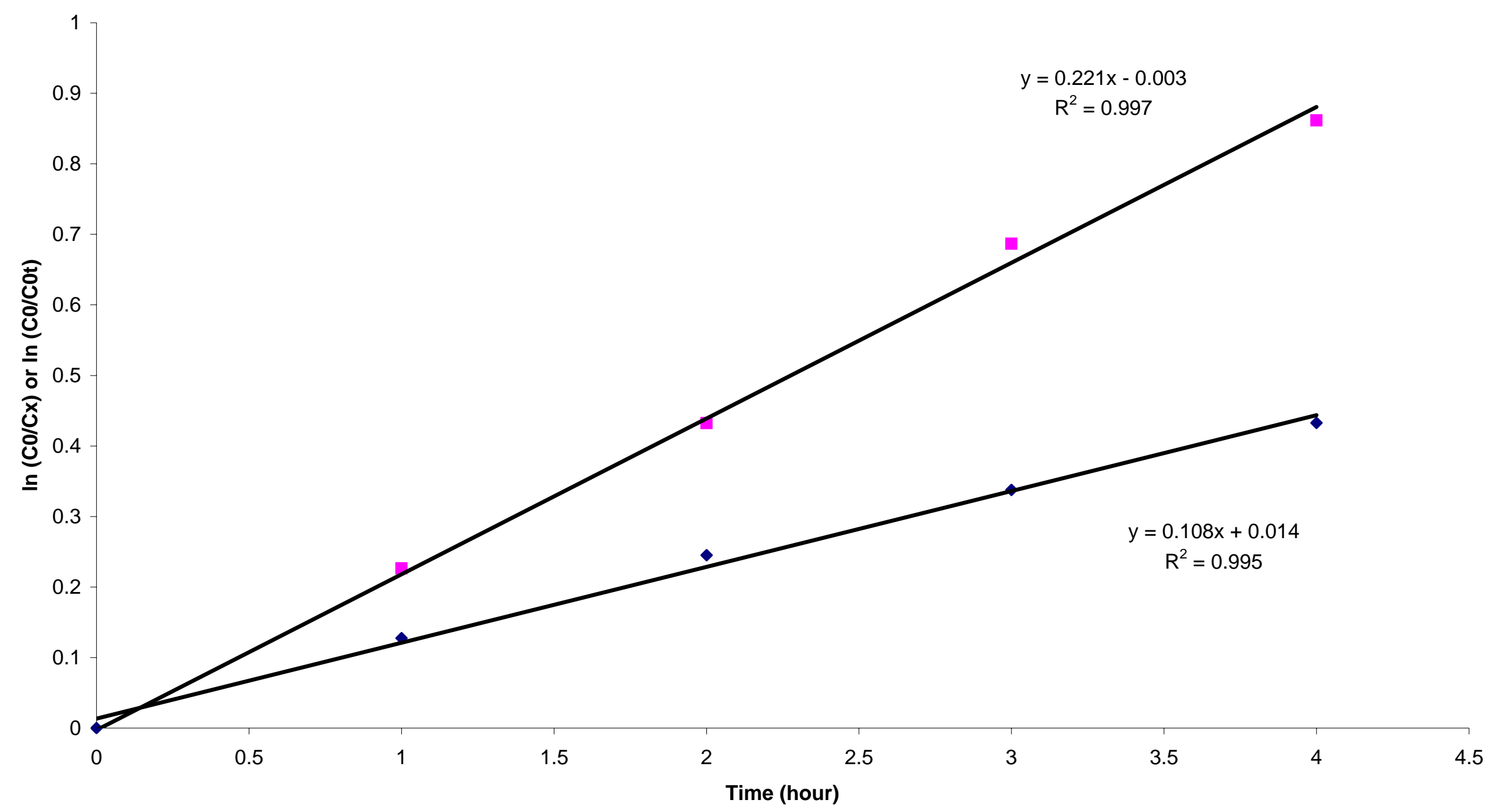




\section{Ester 1 and 1.6\% n-PrOH}

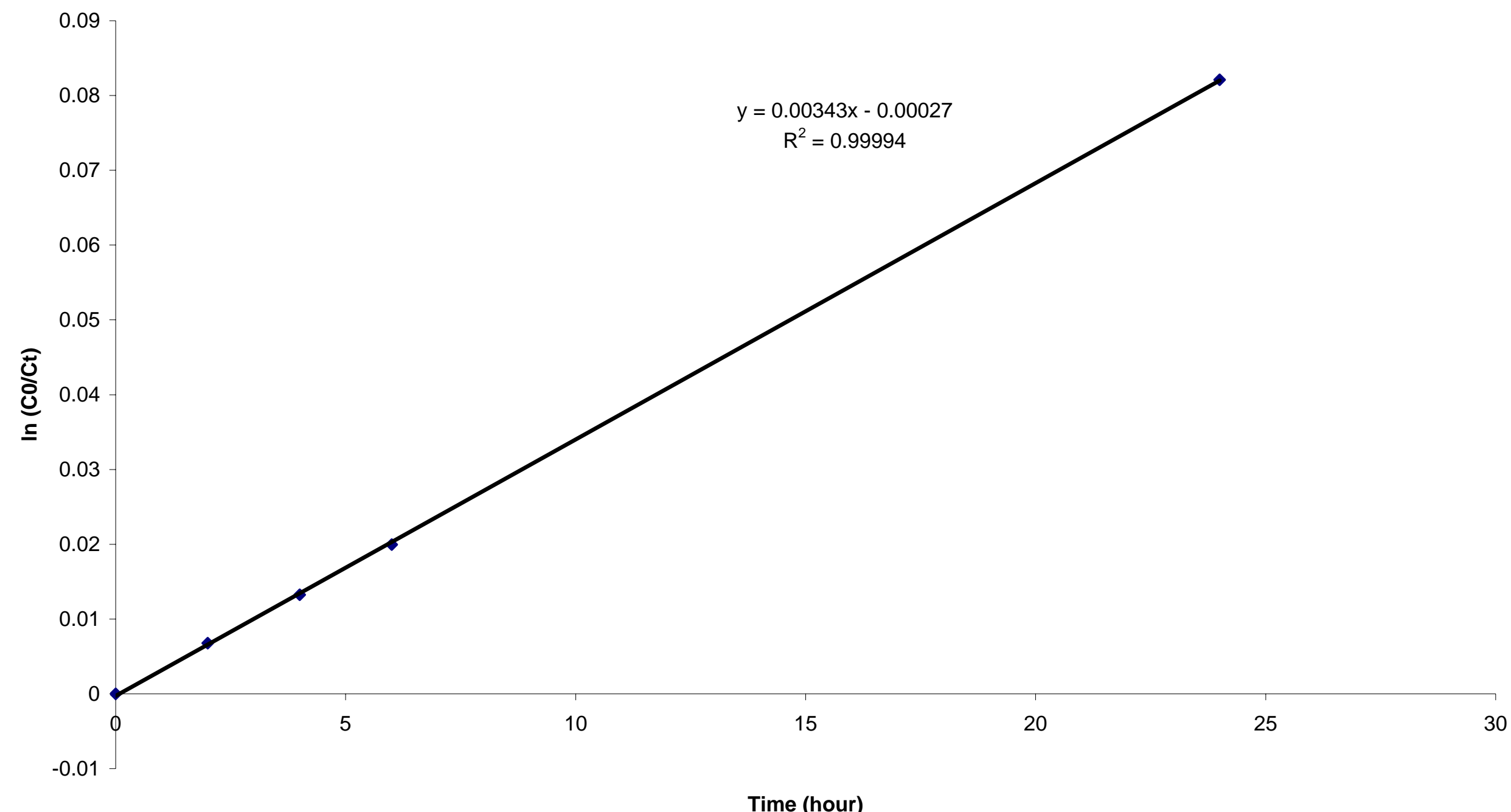




\section{Ester 1 and $1.6 \%$ n-PrOH}

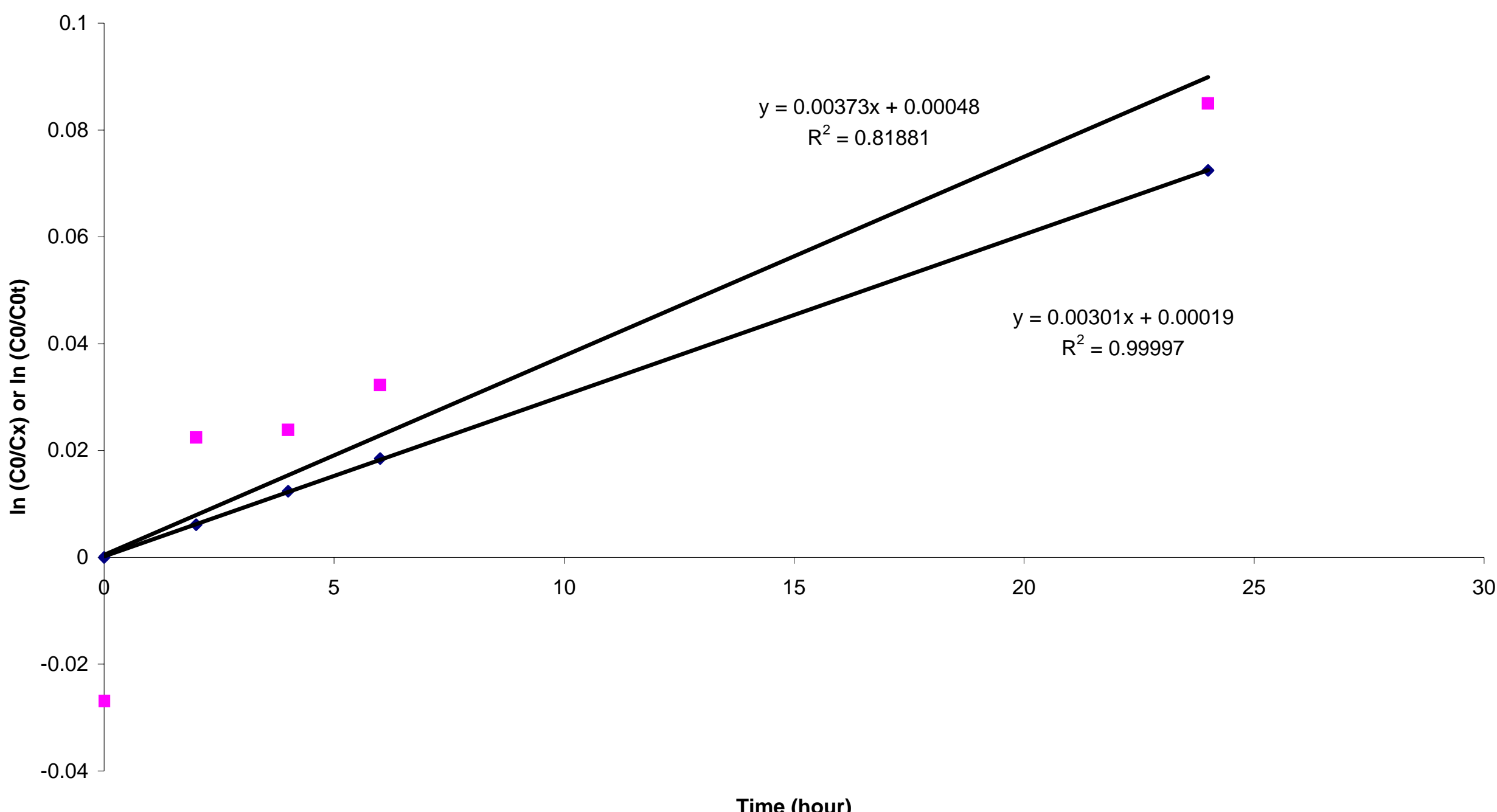




\section{Ester 1 and $0.064 \%$ n-PrOH}

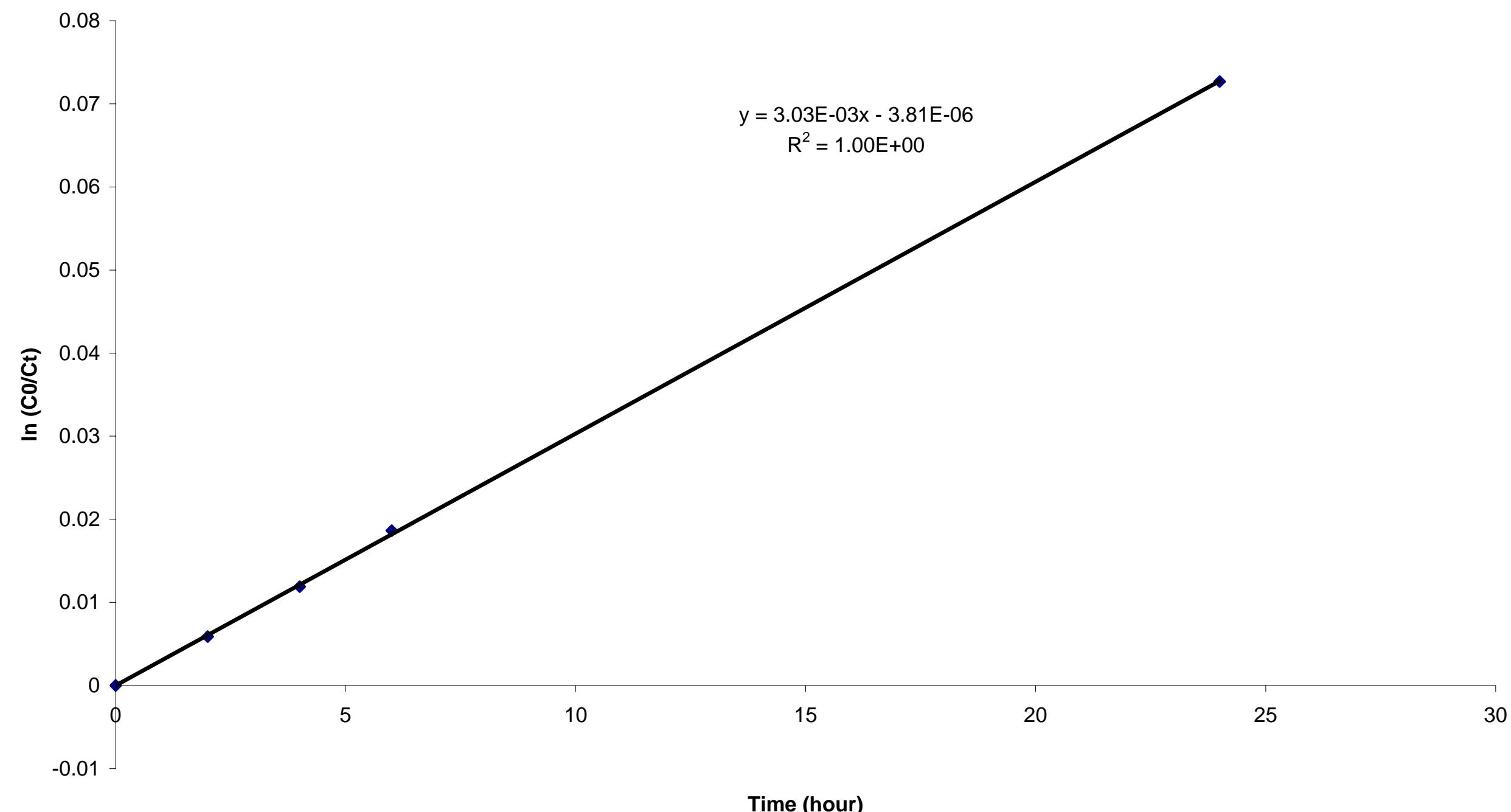




\section{Ester 1 and $0.064 \%$ n-PrOH}

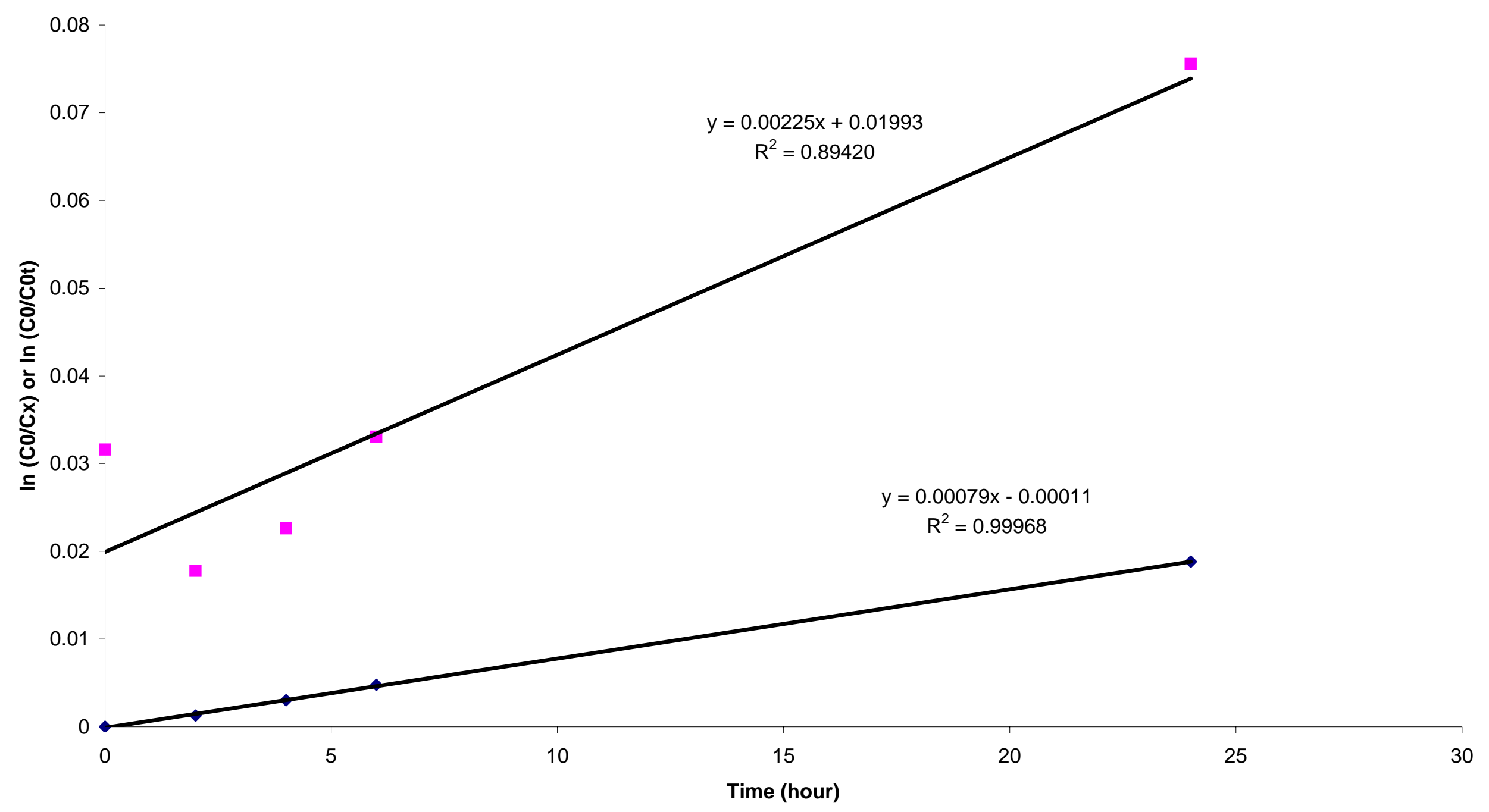


Computational Chemistry. The semi-empirical method pm3 was used for initial conformational search. Up to a dozen conformations for each tautomer was then used as input for $a b$ initio RHF geometry optimizations. After final geometry optimization at the level RHF/6-31G**, single point MP2 calculations using the same basis set was made. All calculations were performed using the Spartan 04 (W) program.

$\underline{\mathrm{XYZ} \text { coordinates (Angstroms) of Compound 1, Energy: }-800858.642 \mathrm{kcal} / \mathrm{mol}}$

\begin{tabular}{lrrr}
$\mathrm{C}$ & 0.2529232 & -1.2590072 & -0.0017059 \\
$\mathrm{C}$ & -1.5893163 & 0.3480576 & 0.0002194 \\
$\mathrm{~N}$ & -0.6606837 & 1.3671982 & -0.0006173 \\
$\mathrm{C}$ & -1.1843294 & -1.0021827 & -0.0006920 \\
$\mathrm{C}$ & 2.5981358 & -0.5730248 & -0.0011103 \\
$\mathrm{C}$ & 1.1631204 & -0.2251988 & -0.0016727 \\
$\mathrm{O}$ & 2.9857415 & -1.7235835 & -0.0032750 \\
$\mathrm{O}$ & 1.4531802 & 2.1141371 & -0.0053535 \\
$\mathrm{C}$ & 0.7180804 & 1.1667590 & -0.0025606 \\
$\mathrm{O}$ & 0.6179661 & -2.5039225 & -0.0021787 \\
$\mathrm{H}$ & 1.5827274 & -2.5544829 & -0.0032207 \\
$\mathrm{C}$ & -2.9553896 & 0.6648034 & 0.0016241 \\
$\mathrm{H}$ & -3.2785773 & 1.6831916 & 0.0024537 \\
$\mathrm{C}$ & -3.8943792 & -0.3309686 & 0.0020469 \\
$\mathrm{H}$ & -4.9382751 & -0.0726195 & 0.0031123 \\
$\mathrm{C}$ & -2.1904750 & -1.9977728 & -0.0002073 \\
$\mathrm{C}$ & -3.5226781 & -1.6673676 & 0.0011501 \\
$\mathrm{H}$ & -4.2615551 & -2.4439255 & 0.0014936 \\
$\mathrm{O}$ & 3.4397729 & 0.4194457 & 0.0025883 \\
$\mathrm{C}$ & 4.8229592 & 0.1050851 & 0.0029312 \\
$\mathrm{H}$ & 5.0832337 & -0.4647896 & 0.8835299 \\
$\mathrm{H}$ & 5.3304156 & 1.0566352 & 0.0052507 \\
$\mathrm{H}$ & 5.0847221 & -0.4612567 & -0.8795082 \\
$\mathrm{C}$ & -1.1159911 & 2.7550607 & -0.0007859 \\
$\mathrm{H}$ & -0.2478709 & 3.3859899 & -0.0025886 \\
$\mathrm{H}$ & -1.7054998 & 2.9621419 & 0.8843180 \\
$\mathrm{H}$ & -1.7084092 & 2.9612387 & -0.8841813 \\
$\mathrm{C}$ & -1.8795489 & -3.7096416 & -0.0010602 \\
& & & \\
\hline & & &
\end{tabular}


XYZ coordinates (Angstroms) of Compound 2, E-conformation, Energy: -957541.140 $\underline{\mathrm{kcal} / \mathrm{mol}}$

$\begin{array}{lrrr}\mathrm{C} & -1.2165769 & -1.6816156 & -0.2458119 \\ \mathrm{C} & -2.8396425 & 0.1430687 & -0.3397735 \\ \mathrm{~N} & 2.1903625 & -0.6388018 & -0.6544230 \\ \mathrm{~N} & -1.9045791 & 0.8911053 & -1.0319812 \\ \mathrm{C} & -2.5488234 & -1.1730607 & 0.0761259 \\ \mathrm{C} & 1.1130948 & -1.3966466 & -0.9440198 \\ \mathrm{C} & -0.2645363 & -0.8511016 & -0.7516912 \\ \mathrm{C} & 2.5723167 & 1.7489593 & -0.3286592 \\ \mathrm{H} & 2.8500776 & 1.8231345 & -1.3630463 \\ \mathrm{C} & 3.5153690 & -1.1922335 & -0.9634359 \\ \mathrm{H} & 4.1719657 & -0.3539890 & -1.1585291 \\ \mathrm{H} & 3.4272767 & -1.7653179 & -1.8737457 \\ \mathrm{C} & 1.7635603 & 1.5507414 & 2.3132310 \\ \mathrm{H} & 1.4503707 & 1.4658290 & 3.3388860 \\ \mathrm{C} & 1.7517257 & 0.4291305 & 1.4967845 \\ \mathrm{H} & 1.4392882 & -0.5218859 & 1.8897223 \\ \mathrm{C} & 2.6006654 & 2.8609562 & 0.4909299 \\ \mathrm{H} & 2.9281339 & 3.8057526 & 0.0943768 \\ \mathrm{C} & 2.1925246 & 2.7662239 & 1.8135521 \\ \mathrm{H} & 2.2104989 & 3.6352261 & 2.4475103 \\ \mathrm{O} & 1.2461583 & -2.5523491 & -1.3119821 \\ \mathrm{O} & 0.1374451 & 1.1085874 & -1.9565446 \\ \mathrm{C} & 2.1478092 & 0.5261996 & 0.1726795 \\ \mathrm{C} & -0.6174432 & 0.4511285 & -1.2864569 \\ \mathrm{O} & -0.9772374 & -2.9459527 & 0.0001861 \\ \mathrm{H} & -0.1589656 & -3.1974737 & -0.4312837 \\ \mathrm{C} & -4.0964095 & 0.6972994 & -0.0684662 \\ \mathrm{H} & -4.3299025 & 1.6938662 & -0.3775951 \\ \mathrm{C} & -5.0427008 & -0.0320549 & 0.6028090 \\ \mathrm{H} & -6.0033066 & 0.4067661 & 0.8057109 \\ \mathrm{C} & -3.5498259 & -1.8829334 & 0.7731332 \\ \mathrm{C} & -4.7780555 & -1.3236413 & 1.0311202 \\ \mathrm{H} & -5.5160055 & -1.8887596 & 1.5657338 \\ \mathrm{C} & -3.3390968 & -3.4924384 & 1.4011599 \\ \mathrm{C} & -2.2695278 & 2.2007041 & -1.5626115 \\ \mathrm{H} & -3.1252756 & 2.1181812 & -2.2214307 \\ \mathrm{H} & -1.4297008 & 2.5748909 & -2.1173357 \\ \mathrm{H} & -2.4990119 & 2.8889658 & -0.7577322 \\ \mathrm{C} & 4.0867931 & -2.0596667 & 0.1526591 \\ \mathrm{H} & 3.4500750 & -2.9168223 & 0.3343914 \\ \mathrm{H} & 4.1901787 & -1.4978918 & 1.0750974 \\ & 5.0709333 & -2.4220799 & -0.1292436\end{array}$


XYZ coordinates (Angstroms) of Compound 2, Z-conformation, Energy: -957537.284 $\underline{\mathrm{kcal} / \mathrm{mol}}$

$\begin{array}{rrrr}\mathrm{C} & 1.5685440 & -1.3351633 & 0.4752502 \\ \mathrm{C} & 3.4008351 & 0.2621721 & 0.2368214 \\ \mathrm{~N} & -1.7143051 & -0.0115553 & -0.1839000 \\ \mathrm{~N} & 2.4791760 & 1.2833603 & 0.3890981 \\ \mathrm{C} & 2.9933862 & -1.0867202 & 0.2642444 \\ \mathrm{C} & -0.7813261 & -0.6401179 & 0.5616032 \\ \mathrm{C} & 0.6708838 & -0.3112484 & 0.4037859 \\ \mathrm{C} & -3.6635903 & 0.9250552 & 0.9127292 \\ \mathrm{H} & -3.0659045 & 1.7855836 & 1.1554567 \\ \mathrm{C} & -1.4413208 & 0.8275235 & -1.3531756 \\ \mathrm{H} & -0.3858722 & 0.7809326 & -1.5614002 \\ \mathrm{C} & -5.1602323 & -1.3214469 & 0.3027761 \\ \mathrm{H} & -5.7377067 & -2.1985567 & 0.0694324 \\ \mathrm{C} & -3.8317672 & -1.2535538 & -0.0777713 \\ \mathrm{H} & -3.3692254 & -2.0716901 & -0.5982160 \\ \mathrm{C} & -4.9956585 & 0.8569913 & 1.2890448 \\ \mathrm{H} & -5.4420444 & 1.6766174 & 1.8239525 \\ \mathrm{C} & -5.7454366 & -0.2653829 & 0.9837295 \\ \mathrm{H} & -6.7788103 & -0.3204122 & 1.2776156 \\ \mathrm{O} & -1.0926033 & -1.5250992 & 1.3390506 \\ \mathrm{O} & 0.3535333 & 1.9966962 & 0.5896574 \\ \mathrm{C} & -3.0838820 & -0.1278545 & 0.2258316 \\ \mathrm{C} & 1.1144960 & 1.0690782 & 0.4571722 \\ \mathrm{H} & -1.6763120 & 1.8585499 & -1.1230637 \\ \mathrm{O} & 1.2011586 & -2.5668694 & 0.7124194 \\ \mathrm{H} & 0.2986012 & -2.5615225 & 1.0415713 \\ \mathrm{C} & 4.7540256 & 0.5776754 & 0.0669408 \\ \mathrm{H} & 5.0772722 & 1.5965656 & 0.0447772 \\ \mathrm{C} & 5.6815529 & -0.4202541 & -0.0818782 \\ \mathrm{H} & 6.7175671 & -0.1641315 & -0.2140570 \\ \mathrm{C} & 3.9795907 & -2.0806699 & 0.0899566 \\ \mathrm{C} & 5.3034972 & -1.7540081 & -0.0774553 \\ \mathrm{H} & 6.0287418 & -2.5322389 & -0.2111108 \\ \mathrm{C} & 3.6332816 & -3.7838734 & 0.0221977 \\ \mathrm{C} & 2.9375442 & 2.6651923 & 0.4982263 \\ \mathrm{H} & 3.6502285 & 2.7616599 & 1.3076216 \\ \mathrm{H} & 2.0814489 & 3.2802202 & 0.7028753 \\ \mathrm{H} & 3.3971267 & 2.9935455 & -0.4269045 \\ \mathrm{C} & -2.2019788 & 0.3657162 & -2.5928193 \\ \mathrm{H} & -1.9610796 & -0.6638383 & -2.8381250 \\ \mathrm{H} & -1.9184502 & 0.9892991 & -3.4352762 \\ \mathrm{H} & -3.2749854 & 0.4437730 & -2.4686850\end{array}$




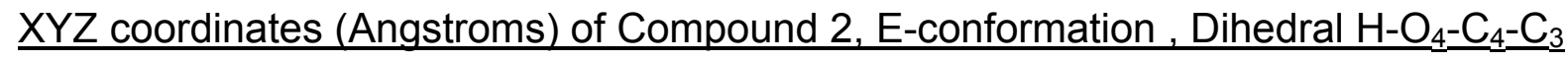
= 90 degrees, Energy: $-957529.149 \mathrm{kcal} / \mathrm{mol}$

\begin{tabular}{rrrr}
$\mathrm{C}$ & 0.7121838 & -0.2055413 & 1.2017590 \\
$\mathrm{C}$ & 2.8925553 & 0.3409575 & 0.2418460 \\
$\mathrm{~N}$ & -2.2597268 & 0.7677690 & 0.3224443 \\
$\mathrm{~N}$ & 2.3087996 & 1.4552525 & -0.3453279 \\
$\mathrm{C}$ & 2.1176334 & -0.5598453 & 1.0032723 \\
$\mathrm{C}$ & -1.2084572 & 1.3540685 & 0.9531363 \\
$\mathrm{C}$ & 0.1836409 & 0.8699396 & 0.6199293 \\
$\mathrm{C}$ & -1.6400544 & 0.1109661 & -1.9480738 \\
$\mathrm{H}$ & -1.3617427 & 1.1295695 & -2.1468363 \\
$\mathrm{C}$ & -3.6008765 & 1.2942231 & 0.6050193 \\
$\mathrm{H}$ & -4.2253450 & 1.0473507 & -0.2442695 \\
$\mathrm{H}$ & -3.5251968 & 2.3714774 & 0.6604847 \\
$\mathrm{C}$ & -2.3710383 & -2.5089789 & -1.4103168 \\
$\mathrm{H}$ & -2.6628698 & -3.5225245 & -1.1984795 \\
$\mathrm{C}$ & -2.4801734 & -1.5409609 & -0.4239140 \\
$\mathrm{H}$ & -2.8630517 & -1.7968331 & 0.5482987 \\
$\mathrm{C}$ & -1.5225882 & -0.8610190 & -2.9273642 \\
$\mathrm{H}$ & -1.1523502 & -0.5917139 & -3.9007711 \\
$\mathrm{C}$ & -1.8874885 & -2.1713877 & -2.6626252 \\
$\mathrm{H}$ & -1.7995659 & -2.9220048 & -3.4278307 \\
$\mathrm{O}$ & -1.3505511 & 2.2272577 & 1.7594714 \\
$\mathrm{O}$ & 0.4607108 & 2.6474558 & -0.8612875 \\
$\mathrm{C}$ & -2.1101441 & -0.2281257 & -0.6862265 \\
$\mathrm{C}$ & 0.9650780 & 1.7393436 & -0.2517110 \\
$\mathrm{O}$ & -0.0592497 & -0.9429306 & 2.0292712 \\
$\mathrm{H}$ & -0.5113960 & -1.6248195 & 1.5539601 \\
$\mathrm{C}$ & 4.2589062 & 0.1062991 & 0.0598595 \\
$\mathrm{H}$ & 4.8578973 & 0.7875925 & -0.5062220 \\
$\mathrm{C}$ & 4.8494386 & -1.0059377 & 0.6039441 \\
$\mathrm{H}$ & 5.9013489 & -1.1734515 & 0.4569739 \\
$\mathrm{C}$ & 2.7614182 & -1.7013734 & 1.5162855 \\
$\mathrm{C}$ & 4.1048350 & -1.9228479 & 1.3274807 \\
$\mathrm{H}$ & 4.5604238 & -2.8032532 & 1.7362626 \\
$\mathrm{C}$ & 1.9322186 & -2.9579078 & 2.4003381 \\
$\mathrm{C}$ & 3.1198685 & 2.3652666 & -1.1488270 \\
$\mathrm{H}$ & 3.9163792 & 2.7887204 & -0.5499060 \\
$\mathrm{H}$ & 2.4801747 & 3.1548871 & -1.4969316 \\
$\mathrm{H}$ & 3.5446784 & 1.8491937 & -2.0017027 \\
$\mathrm{C}$ & -4.2134461 & 0.7550220 & 1.8930273 \\
$\mathrm{H}$ & -3.5810353 & 0.9862323 & 2.7404019 \\
$\mathrm{H}$ & -4.3571764 & -0.3199722 & 1.8474040 \\
$\mathrm{H}$ & -5.1846653 & 1.2125840 & 2.0577532 \\
& & & \\
\hline
\end{tabular}


$\underline{X Y Z \text { coordinates (Angstroms) of Compound 17, Energy: -908377.306 kcal } / \mathrm{mol}}$

\begin{tabular}{lrrr}
$\mathrm{C}$ & 1.3498679 & -1.3477963 & 0.0023929 \\
$\mathrm{C}$ & 3.0937329 & 0.3681569 & -0.0007715 \\
$\mathrm{~N}$ & -1.9959991 & 0.1452923 & -0.0015550 \\
$\mathrm{~N}$ & 2.0991613 & 1.3298263 & -0.0032798 \\
$\mathrm{C}$ & 2.7693514 & -1.0016823 & 0.0023728 \\
$\mathrm{C}$ & -1.0522472 & -0.8018338 & 0.0003528 \\
$\mathrm{C}$ & 0.3776581 & -0.3695239 & 0.0000262 \\
$\mathrm{C}$ & -4.1314618 & 1.1840475 & 0.0099324 \\
$\mathrm{H}$ & -3.6133715 & 2.1276753 & 0.0177305 \\
$\mathrm{C}$ & -5.4593505 & -1.2250043 & -0.0093079 \\
$\mathrm{H}$ & -5.9698049 & -2.1723208 & -0.0169663 \\
$\mathrm{C}$ & -4.0725606 & -1.2167023 & -0.0105400 \\
$\mathrm{H}$ & -3.5276894 & -2.1352478 & -0.0187994 \\
$\mathrm{C}$ & -5.5115794 & 1.1584045 & 0.0105979 \\
$\mathrm{H}$ & -6.0570610 & 2.0857624 & 0.0188773 \\
$\mathrm{C}$ & -6.1883255 & -0.0505188 & 0.0010511 \\
$\mathrm{H}$ & -7.2634095 & -0.0743937 & 0.0017864 \\
$\mathrm{O}$ & -1.3218415 & -1.9978396 & 0.0022532 \\
$\mathrm{O}$ & -0.0524446 & 1.9417390 & -0.0042979 \\
$\mathrm{C}$ & -3.3984775 & -0.0010759 & -0.0005951 \\
$\mathrm{C}$ & 0.7538150 & 1.0321219 & -0.0025435 \\
$\mathrm{O}$ & 1.0582270 & -2.6073970 & 0.0047788 \\
$\mathrm{H}$ & 0.0875563 & -2.6974223 & 0.0044901 \\
$\mathrm{C}$ & 4.4349239 & 0.7714657 & -0.0015597 \\
$\mathrm{H}$ & 4.6944696 & 1.8080207 & -0.0040465 \\
$\mathrm{C}$ & 5.4340203 & -0.1648004 & 0.0008175 \\
$\mathrm{H}$ & 6.4602025 & 0.1564973 & 0.0000910 \\
$\mathrm{C}$ & 3.8339803 & -1.9341300 & 0.0049284 \\
$\mathrm{C}$ & 5.1433372 & -1.5206930 & 0.0041955 \\
$\mathrm{H}$ & 5.9285767 & -2.2505025 & 0.0062789 \\
$\mathrm{C}$ & 3.6272956 & -3.6602037 & 0.0094914 \\
$\mathrm{C}$ & 2.4666734 & 2.7451470 & -0.0059657 \\
$\mathrm{H}$ & 3.0430965 & 2.9870019 & 0.8784061 \\
$\mathrm{H}$ & 1.5628091 & 3.3233449 & -0.0079027 \\
$\mathrm{H}$ & 3.0443732 & 2.9833886 & -0.8904959 \\
$\mathrm{H}$ & -1.6475042 & 1.0811963 & -0.0022245 \\
& & & \\
\hline
\end{tabular}

